\title{
Spatiotemporal expression of regulatory kinases directs the transition from mitotic growth to cellular morphogenesis
}

\author{
Shuo Yang ${ }^{1}$, Jennifer McAdow ${ }^{1}$, Yingqiu Du ${ }^{1}$, Jennifer Trigg ${ }^{2}$, Paul H. Taghert ${ }^{2}$, and
} Aaron N. Johnson ${ }^{1,3}$

${ }^{1}$ Department of Developmental Biology

${ }^{2}$ Department of Neuroscience

Washington University School of Medicine

St. Louis, MO 63110

${ }^{3}$ Author for correspondence: anjohnson@wustl.edu

Running Title: Bsd activates Polo during myogenesis 


\section{Summary}

Embryogenesis depends on a tightly regulated balance between mitotic growth,

2 differentiation, and morphogenesis. Understanding how the embryo uses a relatively small

3 number of proteins to transition between growth and morphogenesis is a central question of

4 developmental biology, but the mechanisms controlling mitosis and differentiation are

5 considered to be fundamentally distinct. Here we show the mitotic kinase Polo, which regulates

6 all steps of mitosis from mitotic entry to cytokinesis [1-3], also directs cellular morphogenesis

7 after cell cycle exit. In mitotic cells, Aurora B (AurB) activates Polo to control a cytoskeletal

8 regulatory module that directs cytokinesis [4-6]. In the post-mitotic mesoderm of late stage

9 embryos, the control of Polo activation transitions to the uncharacterized kinase Back Seat

10 Driver (Bsd), where Bsd activates Polo to direct muscle morphogenesis. The transition between

11 mitotic growth and morphogenesis is accomplished through the spatiotemporal transcriptional

12 regulation of $\mathrm{AurB}$ and Bsd. The functions of Bsd and Polo are conserved, arguing that

13 regulating kinase expression to activate cytoskeletal regulatory modules is a widely used

14 strategy to direct cellular morphogenesis. 


\section{Introduction}

Embryonic development initiates with rapid mitotic divisions, and as development proceeds, cells exit the cell cycle to terminally differentiate and acquire functional morphologies.

17 Tissue patterning through the differential expression of morphogens is a well-understood 18 developmental process [7-11]. Kinases on the other hand are generally considered to be stably

19 expressed mediators of upstream activating proteins [12-16], and most kinases are ubiquitously 20 expressed while the embryo is undergoing rapid cell division (Fig. S1A, Table S1). However, 21 during tissue diversification and organ formation, a majority of kinases show enriched or 22 depleted expression across tissues (Fig. S1A, Table S1). Spatiotemporal transcriptional 23 regulation of kinase function could therefore be a previously unrecognized and essential 24 mechanism that drives the transition from mitotic growth to cellular morphogenesis.

Mitotic growth is controlled by two sets of kinases. Cyclin dependent kinases regulate progression through the cell cycle, and mitotic kinases, which include Aurora kinases and Pololike kinases (Plks), direct mitotic entry, chromosome segregation, and cytokinesis [17-22]. Plk activity is dependent on two conserved protein domains. The C-terminal Polo-Box Domain (PBD) recognizes target substrates, and PBD docking enhances substrate phosphorylation by the Kinase Domain (KD)[22]. Intramolecular interactions between the PBD and the KD dictate the affinity of Polo for specific substrates. The PBD of Drosophila Polo kinase (Polo) binds the microtubule protein Map205 during interphase, which effectively sequesters Polo on

33 microtubules [23]. During mitotic entry, activating phosphorylation of the KD by Aurora B (AurB)

34 relieves PBD binding to Map205 and promotes PBD binding to pro-mitotic substrates and structures [4]. A similar regulatory mechanism has recently been proposed to modulate Polo activity during meiosis [24]. In addition, when Polo is inactive, intramolecular binding between the Polo KD and the PBD masks a nuclear localization signal (NLS). Activating phosphorylation exposes the NLS, allowing Polo to enter the nucleus prior to nuclear envelope breakdown [25]. Although Aurora-mediated Polo activation is an essential regulatory step during cell division, the role of Polo in post-mitotic tissues, if any, is unknown.

Cellular morphogenesis generally initiates in cells that have exited the cell cycle, and

42 gives rise to functionality in highly specialized cells. Cellular guidance is a cytoskeleton-

43 dependent morphogenetic process in which a post-mitotic cell remains spatially fixed and

44 generates long projections that interact with or connect to other cells. Axon guidance is perhaps

45 the most studied form of cellular guidance, and provides the foundation for connecting neurons

46 throughout the nervous system [26]. Nascent myotubes, which are immature post-mitotic

47 muscle precursors, also undergo cellular guidance and extend bipolar projections to connect 
48 with tendon precursors and in turn to the skeleton [27](Movie 1). The body wall muscles in 49 Drosophila are easily visualized in live, unperturbed embryos and have served as an essential

50 model to understand the cellular processes underlying muscle development [28-31], and to

51 uncover the molecular mechanisms that direct cellular morphogenesis [32-37].

52 Here we report a critical function for Polo in the post-mitotic mesoderm, where Polo 53 regulates cellular morphogenesis. Aurora kinases, which are the known Polo activators, are not 54 expressed in the post-mitotic mesoderm. Instead, the uncharacterized serine/threonine (ser/thr) 55 kinase Back seat driver (Bsd) functions in the mesoderm to activate Polo and direct myotube 56 guidance. In addition, the Bsd orthologue Vrk3 activates the Polo orthologue Plk1 in mammalian 57 cells, where Vrk3 and Plk1 both direct muscle morphogenesis. Our studies show the transition 58 from mitotic growth to cellular morphogenesis is achieved through the spatially and temporally 59 restricted expression of the Aurora kinases and Bsd, and that Polo is a conserved effector of 60 Bsd during myotube guidance and cellular morphogenesis.

61 


\section{Results}

63 We carried out a forward genetic screen to identify regulators of myotube guidance, and

64 uncovered a mutation in CG8878 that disrupted muscle development. The body wall muscles in

65 CG8878 embryos showed pronounced navigational defects, so we named the gene back seat

66 driver (bsd) (Figs. 1A-E, S1B). bsd encodes a conserved serine/threonine kinase orthologous to

67 the Vaccinia Related Kinases 3 (VRKs). Proteins in the VRK family contain a single conserved

68 kinase domain (KD) near the N-terminus, and a highly variable C-terminus (Fig. S1C,D).

69 Although VRK proteins have not been shown to regulate myogenesis, pathogenic VRK1

70 variants have been identified in patients with motor neuropathies that may arise from defects in

71 cellular morphogenesis [38-42]. We used an established battery of myogenic assays [37] to

72 visualize muscle development in bsd embryos, and found that Bsd is not required for muscle

73 precursor specification (Fig. S1E,F). Rather, Bsd directs myotube elongation and muscle

74 attachment site selection, which are the two hallmarks of myotube guidance (Fig. 1D-G, Movie

75 1). These studies revealed that Bsd is an essential regulator of cellular morphogenesis in post-

76 mitotic cells.

77 To understand if the spatiotemporal expression of Bsd aligns with the model that kinase expression is differentially regulated during development, we generated and validated an antibody against Bsd (Fig. S2A-C). Bsd is ubiquitously expressed in blastoderm embryos, but

80 after gastrulation Bsd expression in the mitotic mesoderm was significantly reduced (Fig. S2D).

81 As the mesoderm exited the cell cycle and began to diversify, Bsd expression became 82 progressively enriched (Figs. 2A, S2D). Robust Bsd expression continued in the mesoderm 83 during all stages of myotube guidance, but during the final stages of myogenesis Bsd 84 expression in the mesoderm was again reduced (Figs. 2A, S2D,E). The Bsd expression pattern

85 is consistent with the idea that kinase expression is spatially and temporally regulated during 86 embryogenesis.

87 The temporally dynamic expression of Bsd in the mesoderm suggested Bsd functions 88 cell autonomously to regulate muscle morphogenesis. We expressed wild-type Bsd in bsd 89 embryos using the mesodermal driver 24B.Gal4, and found mesodermal Bsd expression 90 dramatically suppressed muscle developmental defects in bsdmutant embryos (Fig. 2B). Since 91 artificially restoring Bsd expression was sufficient to rescue muscle morphogenesis, we asked if 92 Bsd kinase activity in the mesoderm is also necessary for muscle development. Proteins in the 93 VRK family contain a highly conserved ATP binding pocket that is essential for catalytic activity 94 [(Fig. S1C), [43]], and computational structural models predicted that Bsd isoleucine 129 binds 95 ATP (Fig. 2B). Expressing Bsd.I129A in the mesoderm did not suppress the bsd muscle 
96 phenotype (Fig. 2B), which showed Bsd kinase activity is required for proper muscle

97 morphogenesis.

98 To uncover the critical effectors of Bsd during myotube guidance, we used Affinity 99 Purification and Mass Spectrometry (AP-MS) to identify Bsd interacting proteins from whole 100 embryo lysates (Figs. 3A, S3A). To validate the AP-MS results, we screened for protein-protein 101 interactions between Bsd and several candidate proteins in S2 cells. We found a strong, 102 reproducible interaction between full length Bsd and the mitotic kinase Polo (Fig. 3B,C). 103 Interestingly, the truncated Bsd.Q545* protein, which is analogous to the bsd allele identified in 104 our genetic screen, did not physically interact with Polo (Fig. 3B,C), suggesting Bsd-Polo 105 interactions are disrupted in bsd embryos.

106 The physical interaction between Bsd and Polo raised the possibility that Polo has an 107 uncharacterized role in regulating cellular morphogenesis in post-mitotic myotubes. We 108 assessed muscle development in polo mutant embryos, and found that hypomorphic polo 109 alleles caused myogenic phenotypes similar to bsd embryos (Figs. 3D,E S3B,C). We quantified 110 myotube guidance defects in bsd polo double mutant embryos, and found that polo did not 111 enhance the bsd phenotype (Fig. 3F-H). Together these results argue Polo is an essential 112 regulator of myotube guidance, and that Bsd and Polo function in a common myogenic pathway.

113 During mitosis, AurB activates Polo by phosphorylating Thr182 in the Kinase Domain 114 (KD), which alters Polo substrate specificity and promotes Polo nuclear translocation [25]. AurB 115 is broadly expressed in the blastula embryos and in the mitotic mesoderm, but prior to the 116 initiation of myotube guidance, AurB expression becomes restricted to the nervous system [44, 117 45]. Vertebrate Aurora A activates the Polo orthologue Plk1 [46], and like AurB, Drosophila AurA 118 expression is excluded from the post-mitotic mesoderm [45]. Since AurB and AurA are not 119 expressed in the post-mitotic mesoderm during muscle morphogenesis, we hypothesized the 120 control of Polo activation transitions to Bsd during myotube guidance. S2 cells transfected with 121 Bsd had significantly more phosphorylated Polo than controls (Figs. 4A, S4A). Kinase dead 122 Bsd.I129A failed to induce Polo phosphorylation (Fig. S4B), arguing Polo phosphorylation 123 depends on Bsd kinase activity. Bsd also promoted Polo nuclear translocation in S2 cells (Fig. 124 4B).

125 To extend these in vitro studies, we assayed Polo activation in whole embryo lysates 126 and found Bsd promoted Polo phosphorylation in vivo (Fig. 4C). In the post-mitotic mesoderm, 127 the amount of activated Polo significantly increased in muscle precursor nuclei at the onset of 128 myotube guidance, which correlated with Bsd temporal enrichment (Fig. S4C). Strikingly, Polo 129 failed to activate in bsd embryos (Figs. 4D,E, S4C). Total Polo protein levels in the mesoderm 
130 were comparable between wild-type and bsd embryos, suggesting Bsd regulates Polo activation 131 and not Polo expression or stability (Fig. S4D). We expressed activated Polo (T182D) in bsd 132 mutant embryos using the muscle-specific driver Mef2.Gal4, which largely mimics Polo 133 activation. Activated Polo dramatically suppressed the bsd myogenic phenotype (Fig. 4F). 134 Taken together, these studies argue that Bsd-mediated Polo activation is necessary and 135 sufficient to direct myotube guidance.

136 How then does Bsd-activated Polo regulate myogenesis? Two Polo effector proteins, the 137 GTPase activating protein Tumbleweed (Tum) and the kinesin microtubule motor protein 138 Pavarotti (Pav), coordinate cytoskeletal dynamics to position furrow formation at the onset of 139 cytokinesis [5, 47-49]. Since myotube guidance requires dramatic cytoskeletal changes, we 140 hypothesized that the mitotic Polo/Tum/Pav cytoskeletal regulatory module is activated in post141 mitotic myotubes to direct guidance. A role for Tum in myotube guidance was suggested by 142 studies showing Tum regulates the microtubule cytoskeleton to direct myotube elongation [34].

143 To extend previous work, we reanalyzed muscle morphogenesis in tum embryos using 144 improved markers and live imaging (Fig. 4G,H; Movie 2). This analysis revealed that Tum 145 directs both myotube elongation and muscle attachment site selection, the two essential yet 146 mechanistically distinct processes of myotube guidance (Fig. 4G,H; Movie 2). Our studies 147 suggest that the effectors of activated Polo during cytokinesis are also effectors in post-mitotic 148 cells.

149 At the onset of myotube guidance, the microtubule cytoskeleton transitions from a 150 cortical organization to linear arrays that parallel the axis of elongation (Movie 3). Live imaging 151 of cytoskeletal dynamics revealed that the microtubule transition was delayed by over $60 \mathrm{~min}$ in 152 bsd myotubes, and that $b s d$ myotubes failed to maintain linear microtubule arrays (Fig. 5A-C, 153 Movie 3). However, the actin cytoskeleton was largely unaffected (Fig. 5B). The microtubule 154 minus-end nucleator $\gamma$-tubulin initiates the assembly of new microtubules [50], and at the onset 155 of myotube elongation $\gamma$-tubulin foci are predominantly localized to the myotube cortex. As the 156 microtubule cytoskeleton transitions to linear arrays, $\gamma$-tubulin foci appear in the internal 157 myotube cytoplasm. In bsd embryos, $\gamma$-tubulin foci failed to accumulate in the myotube 158 cytoplasm, which likely explains the defects in microtubule organization we observed in bsd 159 myotubes (Figs. 5D,E, S5A,B). Bsd is thus an essential regulator of microtubule dynamics, and 160 our data are consistent with a model in which Bsd activates the Polo/Tum/Pav cytoskeletal 161 regulatory module in post-mitotic cells.

162 To understand if the regulatory functions of Bsd are conserved, we used small interfering 163 RNAs (siRNAs) to knock down Vrk1, Vrk2, and Vrk3 during mammalian muscle morphogenesis. 
164 Under culture conditions that promote differentiation, C2C12 cells (immortalized mouse 165 myoblasts) will form nascent myotubes that extensively elongate [51]. C2C12 cells treated with 166 Vrk1 and Vrk2 siRNAs were morphologically similar to control treated cells after 7 days of 167 differentiation (Fig. S6A,B), but C2C12 cells treated with Vrk3 siRNAs showed significantly 168 reduced elongation (Figs. 6A-D, S6A,B). These myogenic assays functionally confirmed our 169 phylogenetic results showing Bsd is most similar to Vrk3 (Fig. S1C). Post-mitotic C2C12 cells 170 treated with the Plk1 inhibitor Volasterib phenocopied C2C12 cells treated with Vrk3 siRNAs 171 (Fig. 6A-D). In addition, Vrk3 physically interacted with Plk1 (Fig. 6E) and promoted activating 172 phosphorylation of Plk1 in HEK293 cells (Fig. 6F). The Bsd orthologue Vrk3 thus activates the 173 Polo orthologue Plk1 in mammalian cells, suggesting the regulatory role of Bsd is highly 174 conserved. 


\section{Discussion}

$175 \quad$ This study identified Bsd as a conserved regulator of Polo activity. Bsd promoted Polo 176 phosphorylation in cultured cells (Fig. 4A), and was required for activating phosphorylation of 177 Polo at T182 in the post-mitotic mesoderm (Fig. 4D). In addition, activated Polo (T182D) 178 rescued the bsd myogenic phenotype (Fig. 5F), which argues the essential function of Bsd 179 during myotube guidance is to activate Polo. The microtubule cytoskeleton reorganizes to drive 180 morphological changes in nascent myotubes, and Bsd directed microtubule dynamics during 181 myotube guidance (Fig. 5A-D). These observations are consistent with a model in which Bsd 182 activates a Polo/Tum/Pav cytoskeletal regulatory module to direct cellular morphogenesis (Fig. $1836 \mathrm{G})$.

184 Polo is well known as a regulator of cell division. During cytokinesis, the Polo/Tum/Pav 185 cytoskeletal regulatory module uses cortical microtubules to position the contractile ring and 186 initiate furrow formation $[5,52,53]$. We show for the first time that Polo performs an essential 187 function in post-mitotic cells. The Polo/Tum/Pav cytoskeletal regulatory module interacts with 188 the microtubule cytoskeleton in post-mitotic myotubes, suggesting microtubules are the major 189 target of this cytoskeletal regulatory module (Fig. S6D). Tum and Pav also regulate axon 190 guidance in post-mitotic neurons [54], which raises the possibility that the Polo/Tum/Pav 191 cytoskeletal regulatory module is widely activated in post-mitotic cells to direct cellular 192 morphogenesis. Our cytoskeletal regulatory module hypothesis predicts that Polo and Plks 193 function in a myriad of cell types across Metazoans to regulate morphogenesis after cell cycle 194 exit.

195 The transition from mitotic growth to cellular morphogenesis is accomplished through the 196 spatial and temporal regulation of kinase expression. AurB is broadly expressed in blastoderm 197 embryos [44, 45], where it presumably activates Polo to initiate mitotic entry and complete the 198 critical steps of mitosis [3]. AurB is not expressed in the post-mitotic mesoderm, where Bsd 199 instead activates Polo to direct myotube guidance (Fig. 4D-F, 6G). Over 50\% of the Drosophila 200 kinases with known embryonic expression patterns transition from ubiquitous expression before 201 gastrulation to tissue specific expression after gastrulation, and an additional $20 \%$ of kinases 202 show spatially restricted expression throughout development (Table S1, Fig. S1A). Zebrafish 203 kinases show similar embryonic expression patterns (Table S1, Fig. S1A). This conserved, 204 dynamic kinase expression argues that the transcriptional regulation of kinase signaling 205 pathways is broadly employed to direct key events of embryogenesis.

206 Why then would the control of Polo activation transition from AurB to Bsd during cellular 207 morphogenesis? AurB and Bsd phosphorylate T182D to activate Polo [24, 25] (Fig. 4D), so Polo 
208 activation itself likely does not explain the need for multiple activating kinases. However, AurB 209 overexpression can promote cell division in the germline [55], suggesting AurB could also drive

210 sustained mitosis in the embryonic mesoderm. One possibility is that AurB expression is 211 attenuated in the mesoderm to promote mitotic exit and initiate terminal differentiation, while

212 Bsd expression is enriched to activate the Polo/Tum/Pav cytoskeletal regulatory module.

$213 \quad$ Murine Vrk3 regulated myotube elongation, physically associated with Plk1, and 214 promoted activating phosphorylation of Plk1 (Fig. 6A-G). The active site in human VRK3 is 215 divergent at three residues, which led to the hypothesis that VRK3 is a pseudokinase [56]. 216 However, subsequent studies show that VRK3 has kinase activity under certain contexts [57], 217 and our studies support an active role for Vrk3 in promoting phosphorylation. In fact, the 218 conservation of Bsd/Vrk3 cellular and molecular functions is so striking that Vrk3 likely regulates 219 Plk1 activity under a variety of developmental and homeostatic contexts.

220 Although a myogenic role for Vrk3 has not been studied in vivo, Plk1 was recently shown 221 to regulate myogenesis in mice. Muscle-specific deletion of Plk1 blocked limb muscle 222 development during embryogenesis and prevented muscle stem cells from activating after injury 223 [58]. However, it remains unclear how Plk1 is regulated during mammalian muscle development, 224 what the targets of Plk1 are in the muscle lineage, and whether Plk1 regulates muscle 225 morphogenesis. Our study highlights the exciting possibility that Vrk3 regulates Plk1 activity in 226 vivo and that the role of Plk1 during mammalian myogenesis extends well beyond cell cycle 227 control.

\section{Acknowledgements}

We thank Helen McNeill and Mayssa Mokalled for critical reading of the manuscript, and the Drosophila community for stocks and reagents. Our LC-MS was performed by the Washington University Proteomics Core. ANJ was supported by NIH R01AR070299. PHT was supported by NIH R01MH067122. 


\section{References}

1. Glover, D.M., I.M. Hagan, and A.A. Tavares, Polo-like kinases: a team that plays throughout mitosis. Genes Dev, 1998. 12(24): p. 3777-87.

2. Joukov, V. and A. De Nicolo, Aurora-PLK1 cascades as key signaling modules in the regulation of mitosis. Sci Signal, 2018. 11(543).

3. Pintard, L. and V. Archambault, A unified view of spatio-temporal control of mitotic entry: Polo kinase as the key. Open Biology, 2018. 8(8): p. 180114.

4. Kachaner, D., et al., Interdomain allosteric regulation of Polo kinase by Aurora B and Map205 is required for cytokinesis. J Cell Biol, 2014. 207(2): p. 201-11.

5. Ebrahimi, S., et al., Polo kinase interacts with RacGAP50C and is required to localize the cytokinesis initiation complex. Journal of Biological Chemistry, 2010. 285(37): p. 28667-28673.

6. Carmena, M., et al., The chromosomal passenger complex activates polo kinase at centromeres. PLoS Biology, 2012. 10(1): p. e1001250.

7. Strigini, M. and S.M. Cohen, Wingless gradient formation in the Drosophila wing. Curr Biol, 2000. 10(6): p. 293-300.

8. Lecuit, T., et al., Two distinct mechanisms for long-range patterning by Decapentaplegic in the Drosophila wing. Nature, 1996. 381(6581): p. 387-93.

9. Heemskerk, J. and S. DiNardo, Drosophila hedgehog acts as a morphogen in cellular patterning. Cell, 1994. 76(3): p. 449-60.

10. Rushlow, C.A., et al., The graded distribution of the dorsal morphogen is initiated by selective nuclear transport in Drosophila. Cell, 1989. 59(6): p. 1165-77.

11. Roth, S., D. Stein, and C. Nüsslein-Volhard, A gradient of nuclear localization of the dorsal protein determines dorsoventral pattern in the Drosophila embryo. Cell, 1989. 59(6): p. 1189-202.

12. Morgan, D.O., Principles of CDK regulation. Nature, 1995. 374(6518): p. 131-4.

13. Tateno, M., Y. Nishida, and T. Adachi-Yamada, Regulation of JNK by Src during Drosophila development. Science, 2000. 287(5451): p. 324-7.

14. Johnson, G.L. and R. Lapadat, Mitogen-activated protein kinase pathways mediated by ERK, JNK, and p38 protein kinases. Science, 2002. 298(5600): p. 1911-2.

15. Saxton, R.A. and D.M. Sabatini, mTOR Signaling in Growth, Metabolism, and Disease. Cell, 2017. 169(2): p. 361-371.

16. Hiratsuka, T., et al., Regulation of ERK basal and pulsatile activity control proliferation and exit from the stem cell compartment in mammalian epidermis. 2020. 117(30): p. 17796-17807.

17. Lénárt, P., et al., The Small-Molecule Inhibitor BI 2536 Reveals Novel Insights into Mitotic Roles of Polo-like Kinase 1. Current Biology, 2007. 17(4): p. 304-315.

18. Liu, D., O. Davydenko, and M.A. Lampson, Polo-like kinase-1 regulates kinetochoremicrotubule dynamics and spindle checkpoint silencing. Journal of Cell Biology, 2012. 198(4): p. 491-499.

19. Cabral, G., et al., Differential Requirements for Centrioles in Mitotic Centrosome Growth and Maintenance. Dev Cell, 2019. 50(3): p. 355-366.e6.

20. Lane, H.A. and E.A. Nigg, Antibody microinjection reveals an essential role for human polo-like kinase 1 (Plk1) in the functional maturation of mitotic centrosomes. J Cell Biol, 1996. 135(6 Pt 2): p. 1701-13. 
21. Liu, X. and R.L. Erikson, Activation of Cdc2/cyclin B and inhibition of centrosome amplification in cells depleted of Plk1 by siRNA. Proc Natl Acad Sci U S A, 2002. 99(13): p. 8672-6.

22. Archambault, V. and D.M. Glover, Polo-like kinases: conservation and divergence in their functions and regulation. Nat Rev Mol Cell Biol, 2009. 10(4): p. 265-75.

23. Archambault, V., et al., Sequestration of Polo kinase to microtubules by phosphopriming-independent binding to Map205 is relieved by phosphorylation at a CDK site in mitosis. Genes Dev, 2008. 22(19): p. 2707-20.

24. Bonner, A.M., S.E. Hughes, and R.S. Hawley, Regulation of Polo Kinase by Matrimony Is Required for Cohesin Maintenance during Drosophila melanogaster Female Meiosis. Current Biology, 2020. 30(4): p. 715-722.e3.

25. Kachaner, D., et al., Coupling of Polo kinase activation to nuclear localization by a bifunctional NLS is required during mitotic entry. Nature communications, 2017. 8(1): p. 1701.

26. McCormick, L.E. and S.L. Gupton, Mechanistic advances in axon pathfinding. Curr Opin Cell Biol, 2020. 63: p. 11-19.

27. Schnorrer, F. and B.J. Dickson, Muscle building; mechanisms of myotube guidance and attachment site selection. Dev Cell, 2004. 7(1): p. 9-20.

28. Lilly, B., et al., Requirement of MADS domain transcription factor D-MEF2 for muscle formation in Drosophila. Science, 1995. 267(5198): p. 688-93.

29. Baylies, M.K. and M. Bate, twist: a myogenic switch in Drosophila. Science, 1996. 272(5267): p. 1481-4.

30. Chen, E.H., et al., Control of myoblast fusion by a guanine nucleotide exchange factor, loner, and its effector ARF6. Cell, 2003. 114(6): p. 751-762.

31. Dohrmann, C., N. Azpiazu, and M. Frasch, A new Drosophila homeo box gene is expressed in mesodermal precursor cells of distinct muscles during embryogenesis. Genes Dev, 1990. 4(12a): p. 2098-111.

32. Kramer, S.G., et al., Switching repulsion to attraction: changing responses to slit during transition in mesoderm migration. Science, 2001. 292(5517): p. 737-40.

33. Schnorrer, F., I. Kalchhauser, and B.J. Dickson, The transmembrane protein Kon-tiki couples to Dgrip to mediate myotube targeting in Drosophila. Dev Cell, 2007. 12(5): p. 751-66.

34. Guerin, C.M. and S.G. Kramer, RacGAP50C directs perinuclear gamma-tubulin localization to organize the uniform microtubule array required for Drosophila myotube extension. Development, 2009. 136(9): p. 1411-21.

35. Johnson, A.N., et al., Post-transcriptional regulation of myotube elongation and myogenesis by Hoi Polloi. Development, 2013. 140(17): p. 3645-56.

36. Williams, J., et al., Noncanonical roles for Tropomyosin during myogenesis. Development, 2015. 142(19): p. 3440-52.

37. Yang, S., et al., FGF signaling directs myotube guidance by regulating Rac activity. Development, 2020. 147(3).

38. Renbaum, P., et al., Spinal muscular atrophy with pontocerebellar hypoplasia is caused by a mutation in the VRK1 gene. Am J Hum Genet, 2009. 85(2): p. 281-9.

39. Stoll, M., et al., Novel motor phenotypes in patients with VRK1 mutations without pontocerebellar hypoplasia. Neurology, 2016. 
40. El-Bazzal, L., et al., Loss of Cajal bodies in motor neurons from patients with novel mutations in VRK1. Hum Mol Genet, 2019. 28(14): p. 2378-2394.

41. Martín-Doncel, E. and A.M. Rojas, VRK1 functional insufficiency due to alterations in protein stability or kinase activity of human VRK1 pathogenic variants implicated in neuromotor syndromes. 2019. 9(1): p. 13381.

42. Greenbaum, L., et al., Identification of a homozygous VRK1 mutation in two patients with adult-onset distal hereditary motor neuropathy. Muscle Nerve, 2020. 61(3): p. 395-400.

43. Shin, J., et al., NMR solution structure of human vaccinia-related kinase 1 (VRK1) reveals the C-terminal tail essential for its structural stability and autocatalytic activity. J Biol Chem, 2011. 286(25): p. 22131-8.

44. Mesilaty-Gross, S., et al., The Drosophila STAM gene homolog is in a tight gene cluster, and its expression correlates to that of the adjacent gene ial. Gene, 1999. 231(1): p. 173-186.

45. Fisher, B., et al., BDGP insitu homepage. 2012.

46. Seki, A., et al., Bora and the kinase Aurora a cooperatively activate the kinase Plk1 and control mitotic entry. Science, 2008. 320(5883): p. 1655-8.

47. Somers, W.G. and R. Saint, A RhoGEF and Rho family GTPase-activating protein complex links the contractile ring to cortical microtubules at the onset of cytokinesis. Developmental Cell, 2003. 4(1): p. 29-39.

48. Gregory, S.L., et al., Cell division requires a direct link between microtubule-bound RacGAP and Anillin in the contractile ring. Current Biology, 2008. 18(1): p. 25-29.

49. D'Avino, P.P., et al., RacGAP50C is sufficient to signal cleavage furrow formation during cytokinesis. Journal of Cell Science, 2006. 119(Pt 21): p. 4402-4408.

50. Tillery, M.M.L., et al., Centrosomal and Non-Centrosomal Microtubule-Organizing Centers (MTOCs) in Drosophila melanogaster. 2018. p. E121.

51. Blau, H.M., C.P. Chiu, and C. Webster, Cytoplasmic activation of human nuclear genes in stable heterocaryons. Cell, 1983. 32(4): p. 1171-80.

52. Adams, R.R., et al., pavarotti encodes a kinesin-like protein required to organize the central spindle and contractile ring for cytokinesis. Genes Dev, 1998. 12(10): p. 148394.

53. Carmena, M., et al., Drosophila polo kinase is required for cytokinesis. J Cell Biol, 1998. 143(3): p. 659-71.

54. Goldstein, A.Y., Y.N. Jan, and L. Luo, Function and regulation of Tumbleweed (RacGAP50C) in neuroblast proliferation and neuronal morphogenesis. Proc Natl Acad Sci U S A, 2005. 102(10): p. 3834-9.

55. Mathieu, J., et al., Aurora B and Cyclin B Have Opposite Effects on the Timing of Cytokinesis Abscission in $<$ em $>$ Drosophila $</$ em $>$ Germ Cells and in Vertebrate Somatic Cells. Developmental Cell, 2013. 26(3): p. 250-265.

56. Scheeff, E.D., et al., Structure of the pseudokinase VRK3 reveals a degraded catalytic site, a highly conserved kinase fold, and a putative regulatory binding site. Structure, 2009. 17(1): p. 128-38.

57. Park, C.H., et al., Presumed pseudokinase VRK3 functions as a BAF kinase. Biochim Biophys Acta, 2015. 1853(7): p. 1738-48.

58. Jia, Z., et al., A requirement of Polo-like kinase 1 in murine embryonic myogenesis and adult muscle regeneration. 2019. 8. 
59. Nose, A., T. Isshiki, and M. Takeichi, Regional specification of muscle progenitors in Drosophila: the role of the msh homeobox gene. Development, 1998. 125(2): p. 21523.

60. Ranganayakulu, G., et al., Divergent roles for $N K-2$ class homeobox genes in cardiogenesis in flies and mice. Development, 1998. 125(16): p. 3037-3048.

61. Baschal, E.E., et al., Exome Sequencing Identifies a Rare \&lt;em\&gt;HSPG2\&lt;/em\&gt; Variant Associated with Familial Idiopathic Scoliosis. G3: Genes|Genomes|Genetics, 2015. 5(2): p. 167.

62. Hadwiger, G., et al., A monoclonal antibody toolkit for C. elegans. PLoS One, 2010. 5(4): p. e10161.

63. Johannessen, C.M., et al., COT drives resistance to RAF inhibition through MAP kinase pathway reactivation. Nature, 2010. 468(7326): p. 968-72.

64. Agrotis, A. and N. Pengo, Redundancy of human ATG4 protease isoforms in autophagy and LC3/GABARAP processing revealed in cells. 2019. 15(6): p. 976-997.

65. Golsteyn, R.M., et al., Cell cycle analysis and chromosomal localization of human Plk1, a putative homologue of the mitotic kinases Drosophila polo and Saccharomyces cerevisiae Cdc5. J Cell Sci, 1994.107 ( Pt 6): p. 1509-17.

66. Larkin, M.A., et al., Clustal $W$ and Clustal $X$ version 2.0. Bioinformatics, 2007. 23(21): p. 2947-8.

67. $\mathrm{Hu}, \mathrm{J} .$, et al., ATPbind: Accurate Protein-ATP Binding Site Prediction by Combining Sequence-Profiling and Structure-Based Comparisons. J Chem Inf Model, 2018. 58(2): p. 501-510. 


\section{Methods}

\section{Drosophila genetics}

The $b s d^{1}$ allele was recovered in an EMS screen as described [35]. The stocks used in this study include Df(2R)BSC199, Df(2R)BSC699, polo ${ }^{1}$, polo ${ }^{K G 03033}, D f(3 L) B S C 447$, tum $^{D H 15}$, $230 P\{$ UAS-polo.T182D $\}, \quad P\{P T T-G C\}$ polo ${ }^{\text {cc01326 }}$ (Polo-GFP), $\quad P\left\{\right.$ Gal4-tey $\left.{ }^{5053 A}\right\}, \quad P\{G M R 40 D 04-$ 231 GAL4\}attP2 (slou.Gal4), P\{GMR57C12-GAL4\}attP2 (nau.Gal4), P\{Gal4-how $\left.{ }^{24 B}\right\}, P\{U A S-L i f e a c t-$ $232 R F P\}, P\{U A S-e G F P\}$ (Bloomington Stock Center), and $P\left\{G a l 4-k i r r e^{r P 298}\right\}, P\left\{k i r r e^{r P 298} . l a c Z\right\}[59]$, $233 P\{$ Gal4-Mef2 $\}$ [60], and P\{MHC. $\tau$ GFP $\}$ [30]. Cyo, P\{Gal4-Twi\}, P\{2X-UAS.eGFP\}; Cyo,

$234 P\{$ wg.lacZ\}; TM3, $P\{$ Gal4-Twi,$P\{2 X-U A S . e G F P\} ;$ and TM3, $P\{f t z . l a c Z\}$ balancers were used to 235 genotype embryos.

$236 \quad$ Bsd transgenic flies were generated by subcloning the bsd coding sequence (LD23371, Drosophila Genomics Resource Center, supported by NIH grant 2P40OD010949) into pUAStAttb (Kpnl/Xbal). Site directed mutagenesis by PCR sewing was used to make UAS.Bsd.I129A. Plasmids and P(acman) BACs (CH321-61F090 and CH322-02P20) were injected and targeted

240 to a $\phi C 31$ integration site at 22A2 (Rainbow Transgenic Flies; Bloomington Stock 24481); stable 241 insertions were identified by standard methods.

\section{RNA sequencing and variant identification}

Total RNA was collected from 12-24hr embryos per manufacturer's specification

243 (RNeasy kit, Qiagen). cDNA libraries were generated with the TruSeq stranded mRNA sample 244 library kit (Illumina) and sequenced using 50bp paired-end reads on the Illumina HiSeq 2000 245 system. Two technical replicates of $w^{1118}$ and $b s d^{1}$ were prepared and sequenced. Sequence 246 reads were mapped to the Drosophila genome with Genomic Short-Read Nucleotide Alignment 247 Program (GSNAP) using the Cufflinks method. Variants (single nucleotide variants and 248 insertions/deletions) were identified with the Broad Institute's Genome Analysis Toolkit (GATK) 249 as described [61], and the resulting variants were functionally tested by complementation test. 250 The $b s d^{1}$ allele $\left(Q 545^{*}\right)$ was confirmed by Sanger sequencing.

\section{Bsd antibody}

251 We created a fusion Bsd::6xHis fusion protein by PCR, using the C-terminus amino 252 acids 705-1004 of Bsd. We sub-cloned the 598bp fragment into the pHO4d 6xHIS expression 253 vector [62] via conventional restriction enzyme sites. The Bsd 6xHIS fusion construct was 254 transformed into competent BL21 (DE3) pLysS E. coli cells (Invitrogen) and grown, overnight 255 shaking at $37^{\circ} \mathrm{C}$ in DYT supplemented with $100 \mu \mathrm{g} / \mathrm{ml}$ ampicillin. The cells were diluted 25 times 256 in fresh DYT media and grown at $37^{\circ} \mathrm{C}$ to an $\mathrm{OD}_{600}=0.6-0.7$. We added isopropyl $\beta$-D257 thiogalactoside (IPTG) to $1 \mathrm{mM}$ to induce expression of the fusion protein and incubated 
overnight shaking at $18^{\circ} \mathrm{C}$. We purified the 6 XHIS fusion protein on nickel-nitrilotriacetic acid agarose (Qiagen, Valencia, CA) according to the manufacturer's protocols, under native conditions with modified buffers and dialyzed against PBS. We sent the purified protein to Pocono Rabbit Farm \& Laboratory (Canadensis, PA) for guinea pig custom polyclonal antibody

262 production.

\section{Plasmids and mutagenesis}

263 Expression plasmids for the immunoprecipitation screen included the BDGP Flag-HA C264 terminal fusions FMO03130 (Lost), FMO05923 (Map205), FMO06869 (Polo), FMO07294 (Imp), 265 FMO11010 (Yp3), FMO12286 (Jar). Plasmids for expressing tagged proteins in S2 cells were 266 generated by cloning coding sequences into pEntr/SD (Thermofisher, K242020), and 267 recombining the coding sequences into pAc5 promoter destination vectors (pAWM and pAWF).

268 Site directed mutagenesis was performed as described above to generate Bsd.Q545* and 269 Polo.T182A. To generate GST-Bsd for E. coli expression, the bsd coding sequence was 270 subcloned into pGex4T-1 (Sal1/Notl). The Vrk3 mammalian expression construct was 271 generated by recombining pDONR223-VRK3 [Addgene 23687, [63]] into pDEST-CMV-3xFLAG272 EGFP [Addgene 122845, [64]]; pRcCMV-Myc-Plk1 was used without modification [Addgene 273 41160, [65]].

\section{Immunohistochemistry}

$274 \quad$ Antibodies used include $\alpha$-Mef2 (1:1000, gift from R. Cripps), $\alpha$-Tropomyosin (1:600, 275 Abcam, MAC141), $\alpha$-PLK1-phospho-T210 (1:100, Abcam, ab39068), $\alpha$-GFP (1:600, Torrey 276 Pines Biolabs, TP-401), and $\alpha$ - $\beta$ gal (1:100, Promega, Z3781). Embryo antibody staining was 277 performed as described [35]; HRP-conjugated secondary antibodies in conjunction with the TSA 278 system (Molecular Probes) were used to detect primary antibodies. For S2 cell labeling, cells $279\left(5 \times 10^{6}\right)$ were transfected per manufacturer's specifications (Lipofectamine 3000, Invitrogen; 280 applies to all transfections in this study), cultured at $25^{\circ} \mathrm{C}$ in Schneider's Drosophila medium 281 (Sigma, S9895) supplemented with 10\% heat-inactivated fetal bovine serum (FBS, Invitrogen, 282 10082147) for 72h, collected and washed once with S2 medium. Cells were then seeded into a 283 6-well-plate with a glass cover slip and incubated for $1 \mathrm{~h}$. The cells were washed twice with PBS 284 and fixed with 4\% PFA for 15min, and then washed 3 times with PBS. After $1 \mathrm{~h}$ blocking in $28525 \%$ NGS/PBST, cells were incubated with $\alpha$-FLAG antibody (1:1000, Sigma, F3165) and $\alpha$-Myc 286 antibody (1:1000, Sigma, PLA001) in PBST containing $0.5 \% \mathrm{BSA}$ at $4^{\circ} \mathrm{C}$ for $12 \mathrm{~h}$. After five PBS 287 washes, cells were mounted in Vectashield with DAPI $(\mathrm{H}-1000)$.

\section{Imaging and image analysis}


Embryos were imaged with a Zeiss LSM800 confocal microscope; cells were imaged by confocal or with an inverted Zeiss AxioObserver. For time-lapse imaging, dechorionated St12 embryos were mounted in halocarbon oil and scanned at $6 \mathrm{~min}$ intervals. For single-frame live imaging, embryos were dechorionated, mounted in PBT, and directly scanned. Control and mutant embryos were prepared and imaged in parallel where possible, and imaging parameters were maintained between genotypes. Fluorescent intensity and cell morphology measurements

294 were made with ImageJ software.

\section{Affinity Purification and Mass spectrometry}

GST-Bsd and GST was purified from E.coli by standard methods and stored at $-80^{\circ} \mathrm{C}$ in $1 \mathrm{ml}$ aliquots. 12-24hr embryo lysates were collected by homogenizing dechorionated embryos in a Dounce homogenizer with 100 I of lysis buffer $(60 \mathrm{mM}$ Tris pH7.5, 80mM NaCl, 6mM EDTA pH8.0, 2\% Triton X-100, 5mM 1-Naphthyl potassium phosphate, 2mM PMSF, 1X Sigma Phosphatase Inhibitor II, 1X Protease Inhibitor) per $10 \mu \mathrm{l}$ of embryos. The lysate was then centrifuged at 15,000 RCF for $10 \mathrm{~min}$ to pellet large debris. The supernatant was diluted to a final protein concentration of $1 \mathrm{mg} / \mathrm{I}$, aliquoted in $100 \mu \mathrm{l}$ volumes, and flash frozen. For affinity purification, $500 \mu$ l of dialyzed GST-Bsd or GST was bound to $50 \mu$ of PBS washed glutathione sepharose beads (GE Healthcare, 17-0756-01) and incubated at $4^{\circ} \mathrm{C}$ for $30 \mathrm{~min}$. The beads were washed with PBS-1\% Triton X-100. Embryo lysates (100mg protein $/ \mathrm{ml}$ ) were incubated with the protein-bound beads at $4^{\circ} \mathrm{C}$ for $4 \mathrm{hr}$; the beads were then washed three times and submitted to the Washington University Proteomics Core Lab for liquid chromatography-mass spectrometry (1260 Infinity II Bio-Inert LC System, Agilent Technologies).

\section{Immunoprecipitation and Western blotting}

For Drosophila proteins, S2 cells $\left(8 \times 10^{6}\right)$ were transfected with $2 \mu \mathrm{g}$ of each plasmid in 6well plates. Cells were cultured for $48 \mathrm{~h}$, incubated with $2 \mathrm{mM} \mathrm{CuSO}_{4}$ for $24 \mathrm{~h}$ (for FMO plasmids

310 only), collected, washed twice with PBS, lysed with $600 \mu \mathrm{IP}$ buffer (20 mM Hepes, pH=7.4, 150 $311 \mathrm{nM} \mathrm{NaCl}, 1 \% \mathrm{NP} 40,1.5 \mathrm{mM} \mathrm{MgCl}_{2}, 2 \mathrm{mM}$ EGTA, $10 \mathrm{mM} \mathrm{NaF}, 1 \mathrm{mM} \mathrm{Na}_{3} \mathrm{VO}_{4}$, 1X proteinase 312 inhibitor), incubated on ice for $30 \mathrm{~min}$, centrifuged at $12000 \mathrm{Xg}$ for $15 \mathrm{~min}$. The supernatant was

313 collected, incubated with $2 \mu \mathrm{l} \alpha-$ FLAG (Sigma, F3165) or $\alpha$-Myc (PLA001, Sigma) overnight at

$3144^{\circ} \mathrm{C}$, and then incubated with $30 \mu \mathrm{l}$ Dynabeads (Invitrogen, 10007D) for $4 \mathrm{hr}$ at $4^{\circ} \mathrm{C}$. The beads 315 were washed 5X with IP buffer, and immunoblotted with $\alpha$-Myc (1:3000) or $\alpha$-FLAG (1:2000).

316 For in vitro phosphorylation assays, immunoprecipitation was carried out as described, 317 except that anti-Phosphothreonine antibody (1:125, Abcam, ab9337) was used for 318 immunoblotting. For in vivo phosphorylation assays, 200 Polo-GFP and 200 bsd $^{1}$; Polo-GFP 
319 embryos were homogenized in $600 \mu$ IP buffer, large debris was removed by $15 \mathrm{~min}$

320 centrifugation $(12,000 \mathrm{Xg})$, and immunoprecipitation was carried out as described above using

$3212 \mu \mathrm{l} \alpha$-GFP (Torrey Pines Biolabs, TP-401).

322 For mammalian proteins, HEK293T cells were seeded in 6-well plates, grown to 60\% 323 confluency at $37^{\circ} \mathrm{C}$ and $5 \% \mathrm{CO}_{2}$ in Dulbecco's modified Eagle's medium (DMEM; Invitrogen)

324 supplemented with $10 \%$ heat-inactivated FBS. Cells were transfected with $2 \mu \mathrm{g}$ of each plasmid 325 and cultured for 48h. Immunoprecipitations were carried out as described above. Plk1 326 phosphorylation was directly assayed without immunoprecipitation using $\alpha$-PLK1-phospho-T210 327 (1:500, Abcam, ab39068).

328 Western blots were performed by standard method using precast gels (\#456-1096, 329 BioRad), and imaged with the ChemiDoc XRS+ system (BioRad).

\section{siRNA knockdown and inhibitor treatments}

330 For siRNAs, C2C12 cells were seeded in 6-well-plate and grown in standard conditions 331 to $60 \%$ confluency in growth medium (10\% FBS in DMEM), and transfected $10 \mathrm{nM}$ duplexed 332 27nt siRNAs (Integrated DNA Technologies). Transfection efficiency was monitored with Cy3 333 transfection controls (Trifecta Kit, Integrated DNA Technologies). After 24hr, the growth media 334 was changed to differentiation media (2\% horse serum in DMEM). After 7 days differentiation, 335 cells were fixed for $15 \mathrm{~min}$ in $4 \%$ PFA and stained with $\alpha$-alpha-actinin antibody (A7811, Sigma, 336 1:1000).

337 For Volasertib, C2C12 cells were seeded in 6-well-plate and grown in standard 338 conditions to nearly $100 \%$ confluency in growth medium, and treated with $100 \mathrm{nM}$ Volasertib or 339 DMSO for $24 \mathrm{~h}$ in growth medium (No.S2235, Selleck chem). Then the growth medium was 340 changed to differentiation medium (2\% horse serum in DMEM), with 100nM Volasertib or DMSO 341 and incubated for $48 \mathrm{~h}$. Cells were incubated in differentiation medium without Volasertib or 342 DMSO for additional 5 days, fixed and stained as described above.

\section{Quantitative real time PCR}

Total RNA was extracted with RNeasy mini kit (74104, Qiagen), and quantified

344 (Nanodrop 2000). The cDNA was prepared by reverse transcription with M-MLV Reverse 345 Transcriptase (28025013, Thermo) with 2000ng RNA. PowerUp Sybr Green Master Mix 346 (A25742, Thermo) and ABI StepOne system (Applied Biosystems) were used for quantitative 347 RT-PCR. Quantification was normalized to GAPDH. Primers used include:

348 Vrk1-F-5`-ACAGGTTTATGATAATGGACCGC-3`

349 Vrk1-R-5 -CTGGTCAGGGTTCTTGTGACT-3`

350 Vrk2-F-5'-CCGCACATGGACACTCTGTA-3` 
358 performed with DNAMAN (Lynnon Corporation) using the observed divergency distance method.

359 ATPbind [67] was used to predict Bsd ATP binding residues; informatics predictions were 360 compared to the VRK1 ATP binding pocket described in [43].

361 Statistical analyses were performed with GraphPad Prism 8 software, and significance was

362 determined with the unpaired, one-tailed student's t-test or Mann-Whitney test (for non-

363 Gaussain distributions). Gaussian distribution fit curve and skew distribution fit curve were

364 generated wtih Origin 2019 software. Sample sizes are indicated in the figure legends. Data 365 collection and data analysis were routinely performed by different authors to prevent potential 366 bias. All individuals were included in data analysis. 


\section{Figure Legends}

Figure 1. The ser/thr kinase Bsd regulates muscle morphogenesis. (A) Myogenic phenotype in $b s d^{1}$ mutants. Stage 16 embryos labeled with Tropomyosin. Doral Transverse 1 (DT1) and Longitudinal Oblique (LO1) muscles are pseudocolored orange and green. Control $\left(w^{1118}\right)$ embryos showed a stereotypic pattern of body wall muscles. bsd ${ }^{1}$ embryos showed severe defects in muscle morphology, including rounded and bent muscles. The $b s d^{1}$ myogenic defects were completely rescued in embryos harboring one of two overlapping genomic constructs (CH322-02P20 or CH321-61F090). Outcrossing $\mathrm{CH} 321-61 \mathrm{~F} 090$ restored the bsd ${ }^{1}$ myogenic phenotype. (B) Quantification of muscle phenotypes. Individual muscles were scored in segments A2-A8 of St16 embryos; the frequencies of muscle defects are shown as a heat map of the stereotypic muscle pattern in one embryonic segment. (C) Genetic mapping details and the Bsd protein domains. Two overlapping deficiencies failed to complement $b s d^{1}$ (dashed lines indicate breakpoints outside the genomic region shown; see Fig. S1 for transheterozygous phenotypes). The Bsd protein has one kinase domain (KD; orange) that is conserved among the VRK protein family; the Bsd KD is divided by a unique linker (green). The position of the conserved ATP binding pocket is also shown (purple). (D) $b s d^{1}$ Ventral Lateral 1 (VL1) muscle phenotype. Stage 16 embryos labeled for $5053>$ GFP (green) and Tropomyosin (violet). $b s d^{1}$ VL1 muscles were rounded (not shown) and made incorrect or incomplete tendon attachments (white arrowheads). (E) $b s d^{1}$ DT1, LO1, and Ventral Transverse 1 (VT1) muscle phenotypes. Stage 16 embryos labeled for slou>GFP (green) and Tropomyosin (violet). bsd ${ }^{1}$ DT1, LO1, and VT1 muscles made incorrect tendon attachments (white arrowheads; see Movie 1) or remained round (blue arrowhead). (F) Histogram of VL1, DT1, LO1, and VT1 phenotypes ( $\mathrm{n} \geq 54$ per muscle). (G) Phenotype quantification. Muscles were individually scored. normal=0, missing=3, elongation defect=2, attachment site defect $=1$. Final muscle score $=$ sum of phenotypic scores/number of embryos. Open circles (wild-type), closed circles (bsd $\left.{ }^{1}\right)$.

\section{Figure 2. Bsd expression in the mesoderm is progressively enriched during muscle} development. (A) $w^{1118}$ embryos immunolabeled for Bsd (green) and Mef2 (violet). Bsd is expressed in Mef2+ body wall muscle precursors at the onset of myotube guidance (Stage 11), and localized to both the cytoplasm (blue arrow) and the nucleus (white arrow). Overall Bsd expression in Mef2+ cells increased during myotube guidance (Stage 12-14), and Bsd showed enhanced nuclear localization. Once myotube guidance was complete (Stage 16), total Bsd expression in the musculature was reduced and Bsd was evenly distributed between the nucleus and the cytoplasm. (B) Bsd kinase activity in the mesoderm directs muscle 
morphogenesis. Control $\left(w^{1118}\right), \quad b s d^{1} / D f(2 R) B S C 199 \quad$ UAS.Bsd, $\quad b s d^{1} / D f(2 R) B S C 199$ 24B.Gal4>Bsd, and $b s d^{1} / D f(2 R) B S C 199$ 24B.Gal4>Bsd.I129A embryos labeled for Tropomyosin. DT1, LO1, and VL1 muscles are pseudocolored orange, green, and violet. $b s d^{1}$ embryos that expressed wild-type Bsd in the mesoderm showed improved muscle morphology; $b s d^{1}$ embryos that expressed catalytically inactive Bsd.I129A showed extensive muscle defects. Quantification of myogenic phenotypes is as described in Fig. 1. A 3-D model of Bsd is shown with the predicted ATP binding pocket residues colored blue and red; I129 is shaded red.

Figure 3. Bsd and Polo kinase participate in a common myogenic pathway. (A) Heat map of Bsd AP-MS results. Proteins from 12-24hr embryo lysates were affinity purified with control or Bsd-bound GST beads. Relative abundance was determined by MS. (B,C) Immunoprecipitation of S2 cell lysates transfected with Bsd, Bsd.Q545*, and Polo. Full length Bsd interacted with Polo in reciprocal experiments, but Bsd.Q545* and Polo did not interact. (D) polo ${ }^{1}$ VL1 muscle phenotype. Stage 16 embryos labeled for 5053>GFP (green) and Tropomyosin (violet). polo ${ }^{1}$ VL1 muscles were rounded (blue arrowhead) and made incorrect or incomplete tendon attachments (white arrowheads). (E) polo ${ }^{1}$ DT1, LO1, and VT1 muscle phenotypes. Stage 16 embryos labeled for slou>GFP (green) and Tropomyosin (violet). polo ${ }^{1}$ LO1 muscles had attachment site defects (white arrowheads). (F) Muscle morphogenesis phenotypes in $b s d^{1}$ polo ${ }^{1}$ double mutants. Stage 16 embryos labeled with Tropomyosin. DT1, LO1, and VL1 muscles are pseudocolored orange, green, and violet. The frequency and severity of muscle morphology defects was comparable between $b s d^{1}$ and $b s d^{1}$ polo embryos. Quantification of muscle phenotypes is as described in Fig. 1. (G) DT1, LO1, and VT1 phenotypes in $b_{s d^{1}}$ polo $^{1}$ double mutant embryos (labeled as in E). The frequency and severity of DT1, LO1, and VT1 phenotypes was comparable between $b s d^{1}$ and $b s d^{1}$ polo embryos. (H) Quantification of DT1, LO1, and VT1 phenotypes.

Figure 4. Bsd activates Polo to regulate myotube guidance. (A) in vitro phosphorylation assays. Polo was immunoprecipitated from S2 cell lysates and blotted with an antiphosphothreonine (pThr) antibody. Cells transfected with Bsd showed significantly more phosphorylated Polo than controls. (B) Polo localization in S2 cells. Bsd.Myc, Polo.Flag, and Polo.T182A.Flag were transfected into S2 cells; transgenic proteins were detected with anti-Myc (red, Bsd) and anti-Flag (green, Polo). Polo localized to the nucleus in a subset of control cells (middle column). The frequency of cells showing nuclear Polo localization was increased when Bsd was co-transfected with Polo (left column and graph). Inactivatable Polo (T182A) did not 
localize to the nucleus (right column and graph). (C) in vivo phosphorylation assay. Endogenous GFP-tagged Polo was immunoprecipitated from embryo lysates and blotted with pThr. bsd ${ }^{1}$ embryo lysates showed significantly less phosphorylated Polo than controls. (D) Stage 12 embryos immunolabeled for activated Polo (Polo ${ }^{\mathrm{pT} 182}$, green) and Mef2 (violet). Myonuclear Polo ${ }^{\text {T182 }}$ levels were reduced in $b s d^{1}$ embryos compared to controls. (E) Polo ${ }^{\text {pT182 }}$ fluorescent intensity in myonuclei. Nuclear Polo ${ }^{\mathrm{p} T 182}$ fluorescence increased in control ( $\left.b s d^{1} / \mathrm{Cyo}\right)$ embryos during Stage 12, but not in $b s d^{1}$ embryos. Control and experimental embryos were derived from the same preparation. (F) Activated Polo rescues the bsd phenotype. bsd ${ }^{1}$ UAS.Polo.T182D and bsd $^{1}$ Mef2.Gal4>Polo.T182D embryos labeled for Tropomyosin. DT1, LO1, and VL1 muscles are pseudocolored orange, green, and violet. Expressing active (phosphomimetic) Polo.T182D in the mesoderm of $b s d^{1}$ embryos suppressed the $b s d^{1}$ myogenic phenotype. Quantification of myogenic phenotypes is as described in Fig. 1. (G) tum ${ }^{D H 15}$ VL1, DT1, LO1, and VT1 muscle phenotypes. Stage 16 embryos labeled for 5053>GFP (green) and Tropomyosin (violet) or slou>GFP (green) and Tropomyosin (violet). tum ${ }^{D H 15}$ muscles showed attachment site defects similar to $b s d^{1}$ muscles. $(H)$ Histogram of VL1, DT1, LO1, and VT1 phenotypes ( $n \geq 54$ per muscle). (e) early, (I) late, (ns) not significant, ${ }^{* *}(p<0.01)$, ${ }^{* * * *}(p<0.0001)$. Error bars represent SEM.

Figure 5. Bsd directs microtubule reorganization during myotube guidance. $(A, B)$ Live imaging stills of LO1 myotubes in Stage 12-15 embryos that expressed Nod.GFP (green, microtubule minus ends) and LifeAct.RFP (violet, F-actin). Transgene expression was controlled by slou.Gal4. Live imaging initiated once Nod.GFP fluorescence was detectable (0min). Control LO1 myotubes showed a linear array of microtubule minus ends at the onset of imaging (white arrowheads). Nod.GFP did not co-localize with F-actin at the myotube leading edges, indicative of microtubules with a minus-ends-in conformation. The linear configuration of microtubule minus ends in $b s d^{1}$ LO1 myotubes was delayed; Nod.GFP remained cortical through the early stages of myotube elongation (blue arrowheads). $b s d^{1}$ microtubule minus ends often overlapped with the F-actin domain (red arrowhead). (B) LO1 myotubes from (A) showing F-actin expression by heat map. Scale bar represents the detection range. F-actin levels were equivalent in control and $b s d^{1}$ myotubes. (C) Quantification of Nod.GFP distribution. GFP fluorescence was traced in each frame of three independent live-imaging experiments per genotype. Nod.GFP localization was stable in control myotubes, with a low roundness score (more linear), and an angle consistent with the final LO1 attachment angle. $b s d^{1}$ myotubes showed fluctuating Nod.GFP localization, with an overall high roundness score (more cortical) 
and an angle that deviates from the control attachment angle. (D) Single confocal scans of multinucleate LO1 myotubes from Stage 12 slou>GFP embryos labeled for $\gamma$-tubulin (green), GFP (violet), and DAPI (blue). Control ( $\left.b s d^{1} / C y o\right)$ myotubes showed both cortical and internal cytoplasmic $\gamma$-tubulin foci, with internal foci concentrated toward the myotube leading edges (red arrowheads). $b s d^{1}$ myotubes had significantly fewer internal $\gamma$-tubulin foci compared to controls, but an equivalent number of cortical $\gamma$-tubulin foci. Heat map scale is the same as in (B). (E) Quantification of $\gamma$-tubulin foci in multinucleate Stage 12 myotubes. Embryos used for $(D, E)$ were derived from the same preparation. (ns) not significant. ${ }^{* *}(p<0.01)$. Error bars represent SEM.

Figure 6. The Bsd orthologue Vrk3 is required for myotube elongation. (A) $\mathrm{C} 2 \mathrm{C} 12$ cells treated with siRNAs against murine Vrk3 or with an inhibitor of Plk1 (Volasterib, 100nM). Cells were fixed after 7 days in differentiation media and labeled for $\alpha$-actinin (green) to detect differentiated myotubes. Vrk3 knockdown and Volasterib treated myotubes were shorter than controls and were often rounded. (B) Quantification of cumulative myotube length. (C) Myotube length distribution. Solid lines show the Gaussian distribution fit curve (blue) and skew distribution fit curve (red). (D) Individual myotubes were traced to determine a roundness score. Vrk3 RNAi and Volasterib treated myotubes showed a higher roundness score, indicating increased circularity. (E) Immunoprecipitation of HEK293 cell lysates showed a physical interaction between Vrk3 and Plk1. (F) Western blot of HEK293 cell lysates transfected with Vrk3 and Plk1. Vrk3 promoted Plk1 phosphorylation. (G) Model showing Polo activation transitions from AurB in mitotic tissues to Bsd in post-mitotic tissues. Polo controls cytoskeletal regulatory module to direct cytokinesis and myotube guidance. ${ }^{* *}(p<0.01),{ }^{* * * *}(p<0.0001)$. Error bars represent SEM. 
Movie Captions.

Movie 1. Bsd regulates myotube guidance. Live imaging of LO1 myotubes from Stage 12 slou>eGFP,nRFP embryos. $b s d^{1}$ myotubes failed to elongate anteriorly and attached at the posterior of the segment. GFP (green), RFP (violet). Arrowheads denote the myotube dorsal leading edge.

Movie 2. Tum regulates myotube guidance. Live imaging of an LO1 myotubes from Stage 12 tum $^{\text {DH15 }}$ slou>eGFP embryos. tum ${ }^{D H 15}$ myotubes often elongated toward the incorrect muscle attachment site.

Movie 3. Microtubule reorganization is delayed in $b_{s d^{1}}$ myotubes. Live imaging of LO1 myotubes from Stage 12 slou>Nod.GFP,LifeAct.RFP embryos. Nod.GFP localizes to microtubule minus-ends; LifeAct localizes to F-actin. The formation of linear minus-end arrays was delayed in $b s d^{1}$ myotubes. GFP (green), RFP (violet).

Table S1. Embryonic expression patterns of Drosophila and zebrafish protein kinases. 


\section{Supplemental Figure Legends}

Figure S1, related to Figure 1. (A) in silico expression analysis of Drosophila and zebrafish protein kinases. Embryonic expression patterns for all kinases were queried in Flybase and Zfin, and those with reported expression patterns were graphed. Expression patterns were subdivided by embryonic stage (Stages 0-5 in Drosophila occur prior to gastrulation), and classified as Tissue/Group Enriched, (meaning the kinase was expressed in a single tissue or a group of tissues but not all tissues), Ubiquitous, or Low Expression (in which the expression pattern was too faint to categorize). No Data indicates the expression pattern is known for only a subset of embryonic stages. See Table S1 for individual expression patterns. (B) bsd deficiency mapping. Live Stage 16 embryos that expressed Myosin Heavy Chain (MHC)-GFP. $b s d^{1} / D f(2 R) B S C 199$ and $b s d^{1} / D f(2 R) B S C 699$ embryos phenocopied $b s d^{1}$ embryos. A control $\left(w^{1118}\right)$ embryo is shown for comparison. (C) Phylogenetic analysis of human, mouse, and fly Vrk proteins. The homology tree was generated using observed divergency as the distance method. The percent homology among species is shown. (D) Protein alignment of the Vrk proteins from (C); black shading shows identical residues in all species, pink shading shows residues that are the same in most species, blue shading shows residues that are the same in at least $50 \%$ of species. The position of the $b s d^{1}$ Q545* mutation is given (red box). Residues in the previously defined Kinase Domain (orange line) and ATP binding pocket (violet) for human Vrk1 are shown. Note that the Bsd kinase domain is split by a unique linker region (green line), and the C-termini of the Vrk proteins show low sequence homology. (E) Stage 12 5053>GFP embryos labeled for GFP. Control and $b s d^{1}$ embryos showed an equivalent number of VL1 founder cells. (F) Stage 12 slou>GFP embryos labeled for GFP. Control and $b s d^{1}$ embryos showed an equivalent number of GFP+ cells. (ns) not significant. Error bars represent SEM.

Figure S2, related to Figure 2. (A-C) Validation of the Bsd antibody. (A) Western blots of control $\left(w^{1118}\right), D f(2 R) B S C 199$, and $r p 298>B s d$ embryo lysates using the Bsd antibody (left) or preimmune serum (right). UAS/Gal4 expressed Bsd was detectable, but we could not detect endogenous Bsd by Western blot. (B) Western blot of S2 cell lysates transfected with control or Bsd.Myc expressing plasmids. The Bsd and Myc antibodies recognized proteins of the same molecular weight. (C) Stage 12 embryos immunolabeled with the Bsd antibody (green) and Mef2 (violet). Signal from the Bsd antibody was not observed in Df(2R)BSC199 body wall muscle precursors. The embryos shown were prepared in parallel. (D) Complete embryonic expression of Bsd. Embryos were immunolabeled as in (C). Multiple confocal projections are shown for each embryo to compare expression in the ectoderm and the mesoderm. Bsd was 
ubiquitously expressed prior to gastrulation (Stage 5), and Bsd expression remained high in the ectoderm after gastrulation. Bsd expression in the mesoderm showed a progressive enrichment from Stage 9 to Stage 14, and then decreased. (E) Quantification of Bsd localization in myonuclei compared to nuclei in non-muscle cells.

Figure S3, related to Figure 3. (A) Coomassie stained control gel for GST immunoprecipitation experiment described in Figure 3. Bsd-bound GST beads (lane 5) recovered a large number of unique bands compared to control GST beads (lane 4). (B) Myogenic phenotypes in polo mutants. Stage 16 embryos labeled with Tropomyosin. DT1, LO1, and VL1 muscles are pseudocolored orange, green, and violet. The polo alleles showed stronger phenotypes in trans to $D f(3 L) B S C 447$, arguing the alleles are hypomorphic. Frequency of muscle phenotypes is as described in Fig. 1. (C) Histogram of polo ${ }^{1}$ VL1 phenotypes $(n=54)$.

Figure S4, related to Figure 4. (A) Control phosphorylation assay. Lysates from S2 cells transfected with a control plasmid or a plasmid expressing Bsd.Myc were immunoblotted with anti-phosphothreonine ( $p$ Thr). Whole lystates showed equivalent global pThr levels. (B) in vitro phosphorylation assay. Polo was immunoprecipitated from S2 cell lysates and blotted with a anti-pThr. Cells transfected with Bsd.I129A showed equivalent phosphorylated Polo as controls. (C) Early and late Stage 12 embryos immunolabeled for activated Polo ${ }^{\text {T182 }}$ (pPolo, green) and Mef2 (violet). Myonuclear Polo ${ }^{\text {pT182 }}$ increased during Stage 12 in control ( $b s d^{1}$ heterozygous) but not $b s d^{1}$ embryos. Control and $b s d^{1}$ embryos were labeled in the same preparation. (D) Stage 12 embryos with an endogenous GFP-tagged Polo $\left(P o l O^{G F P}\right)$ that expressed $r p 298>n L a c Z$ were immunolabeled for GFP (green) and lacZ (red). Fluorescent intensity and cellular localization of total Polo protein was comparable between control and $b s d^{1}$ embryos. Graph shows Polo and nlacZ fluorescent intensity across myoblasts.

Figure S5, related to Figure 5. (A) Mononucleate LO1 myotubes from Stage 12 slou>GFP embryos labeled for $\gamma$-tubulin (green), GFP (violet) and DAPI (blue). Mononucleate, polarized myotubes have just initiated myotube guidance (see Movie 1 ). Control ( $\left.b s d^{1} / C y o\right)$ myotubes showed only cortical $\gamma$-tubulin foci. $b s d^{1}$ myotubes had an equivalent number of cortical $\gamma$-tubulin foci compared to controls. (B) Quantification of $\gamma$-tubulin foci in mononucleate Stage 12 myotubes. Embryos used for $(A, B)$ were derived from the same preparation. (ns) not significant. Error bars represent SEM. 
Figure S6, related to Figure 6. (A) C2C12 cells treated with control (scrambled) siRNA or a cocktail of two siRNAs against murine Vrk1, Vrk2, or Vrk3. Cells were fixed after 7 days in differentiation media and labeled for $\alpha$-actinin (green) to detect differentiated myotubes and DAPI (blue). Vrk3 knockdown myotubes were shorter than controls and often rounded. Vrk1 and Vrk2 knockdown myotubes were indistinguishable from control cells. (B) Quantitative real time PCR of siRNA treated C2C12 cells. Fold change is relative to control treated cells (red line). (C) $\mathrm{QQ}$ plot of myotube distribution, related to Figure $6 C .{ }^{* *}(p<0.01),{ }^{* * *}(p<0.0001)$. Error bars represent SEM. Model of the Polo/Tum/Pav cytoskeletal regulatory module. Since Polo activation unmasks a nuclear localization signal, Bsd likely activates the Polo/Tum/Pav cytoskeletal regulatory module in the cytoplasm. 
Figure 1. The ser/thr kinase Bsd regulates muscle morphogenesis

A

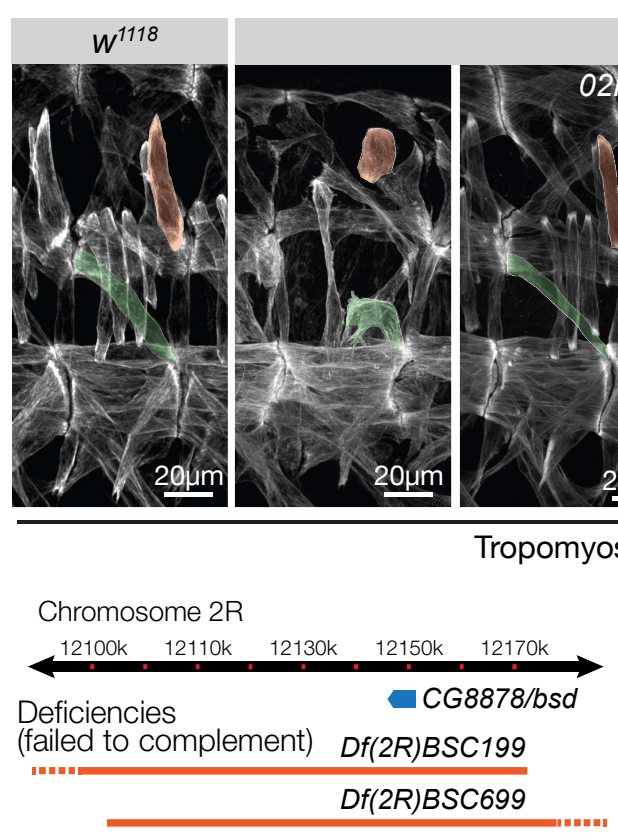

Genomic rescue constructs

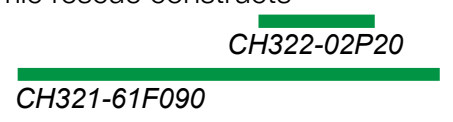

D

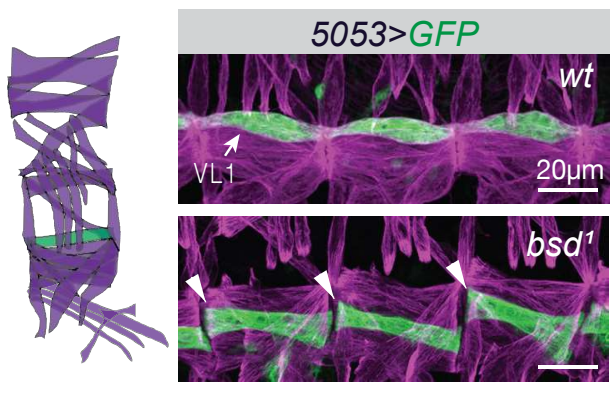

$\mathrm{F}$

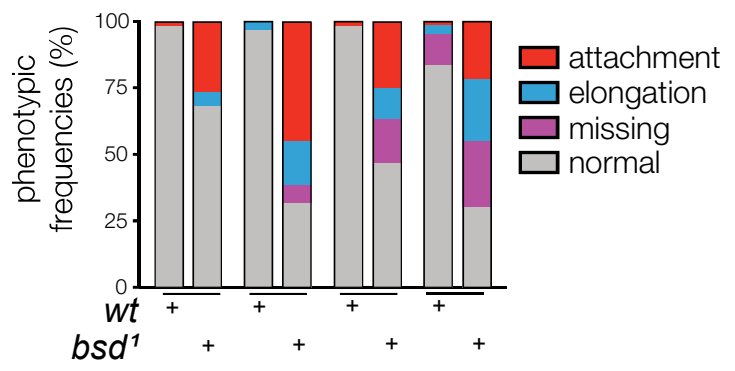

$E$

B

$b s d^{1}$
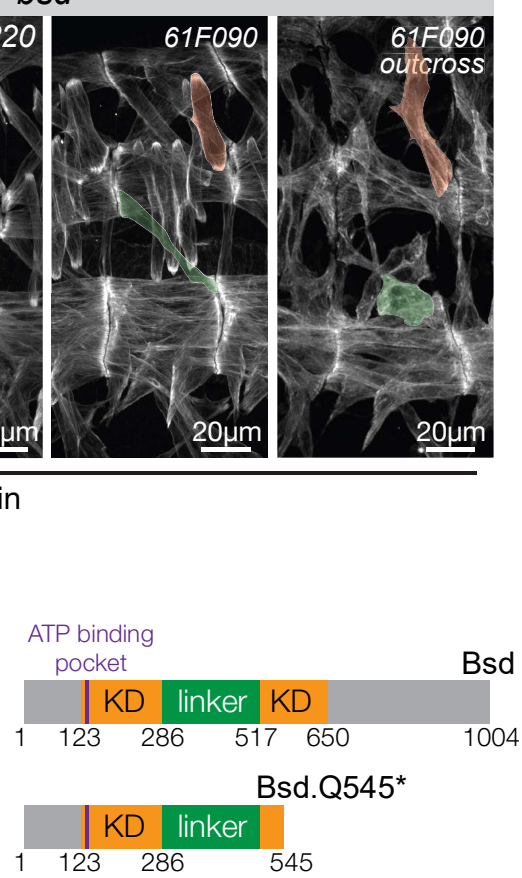

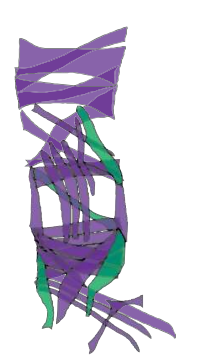

G
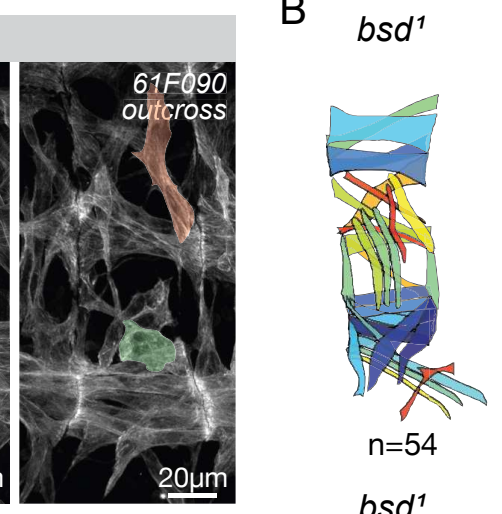

$20 \mu \mathrm{m}$

bsd ${ }^{1}$,

61F090
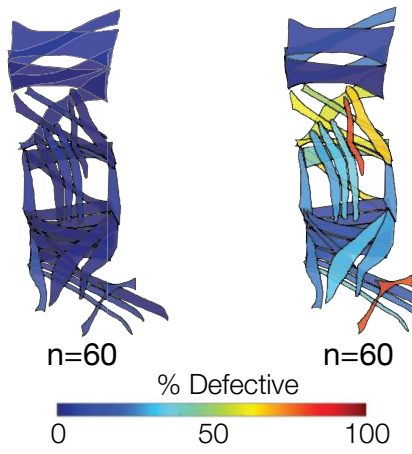

00

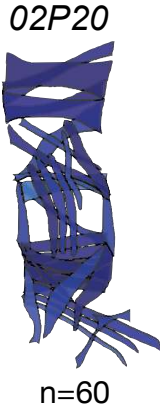

bsd $^{1}, 61 F 090$

outcross

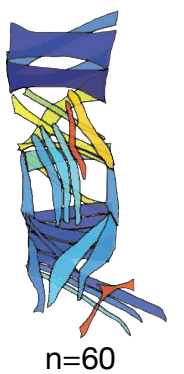

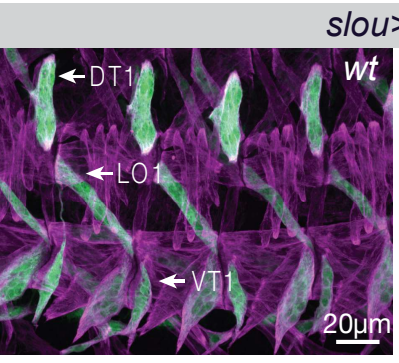

Tropomyosin

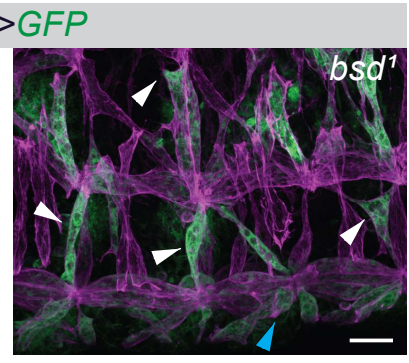

Tropomyosin

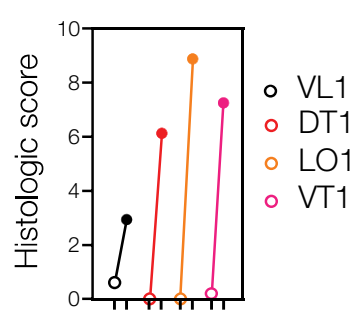


Figure 2. Bsd expression in the mesoderm is progressively enriched during muscle development

A

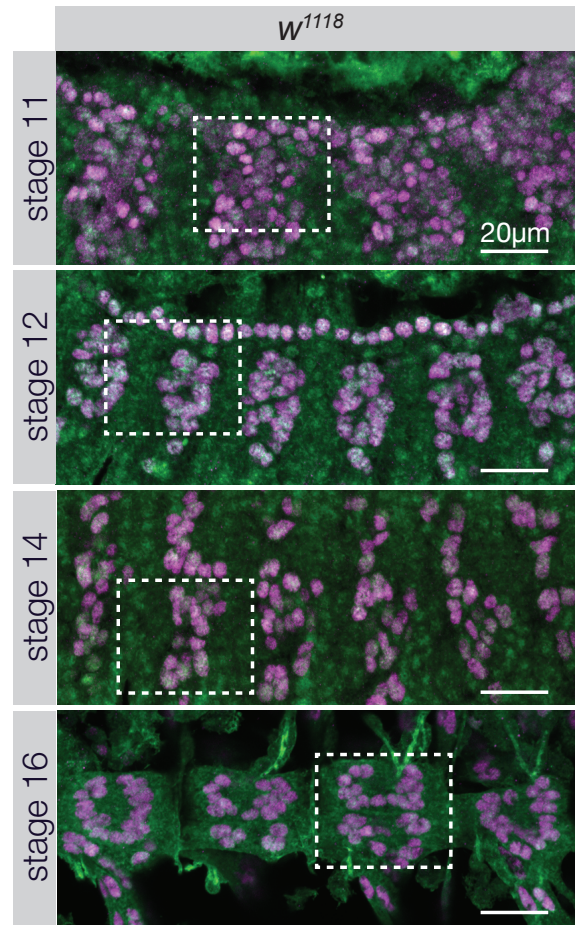

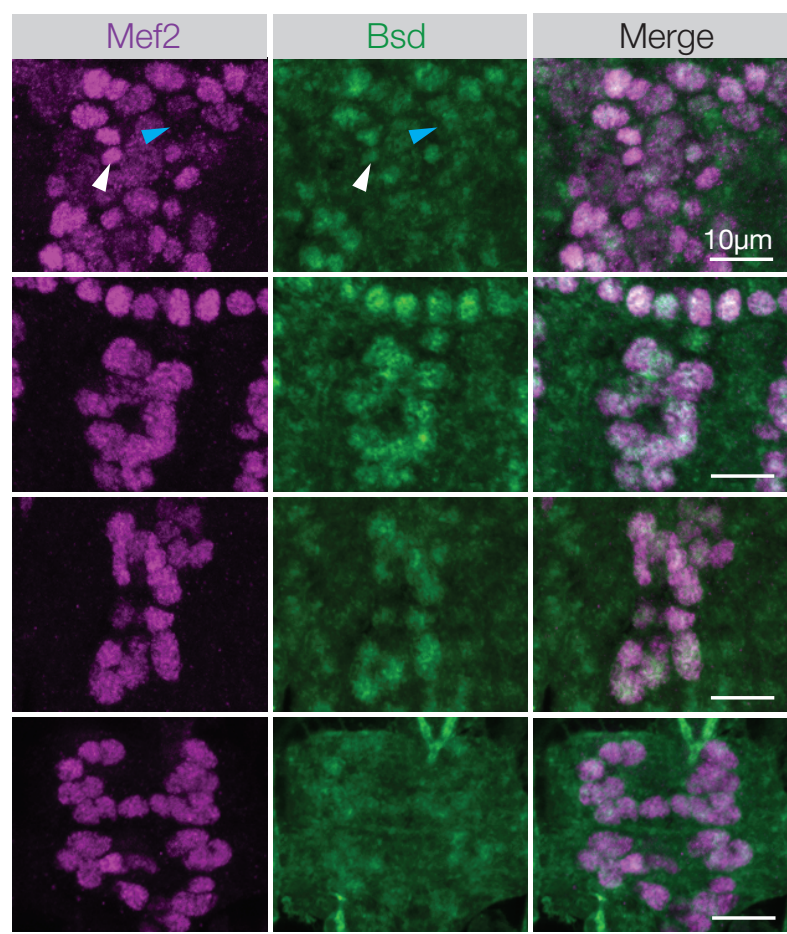

B
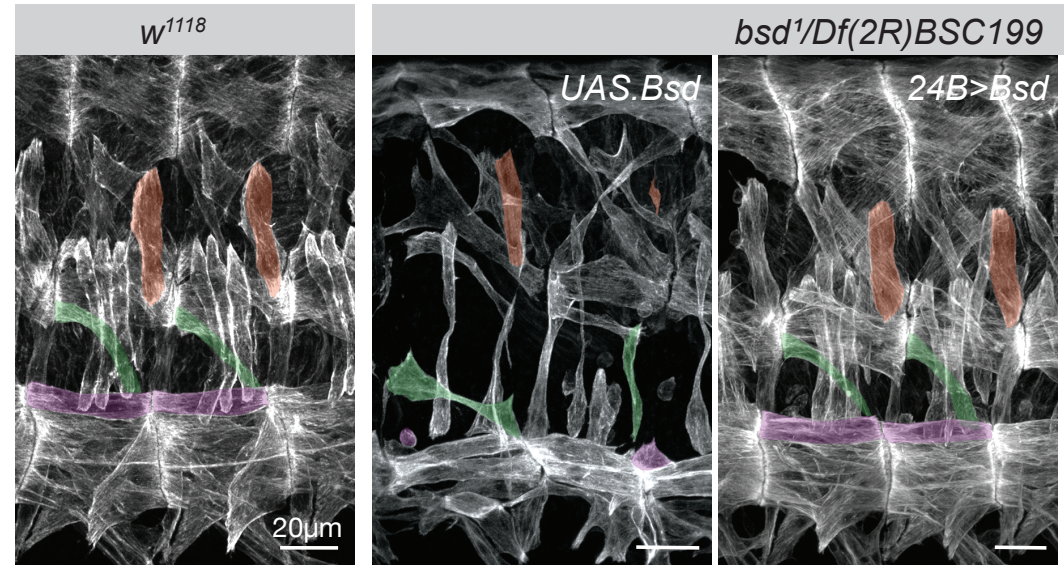

Tropomyosin

$b s d^{1} / D f \quad b s d^{1 / D f} \quad b s d^{1} / D f$

UAS.Bsd $24 B>B s d \quad 24 B>1129 A$
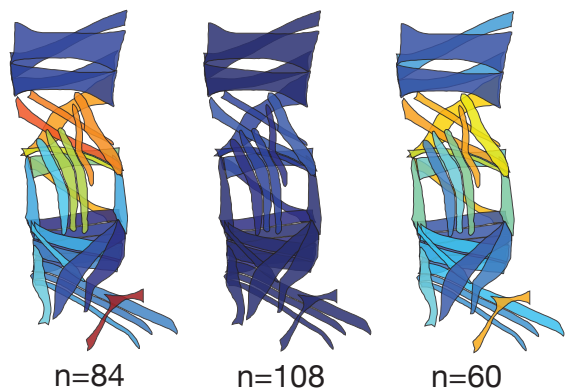

$n=60$

$$
\mathrm{n}=108
$$

$\%$ Defective

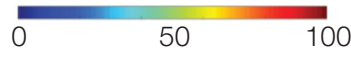

Bsd

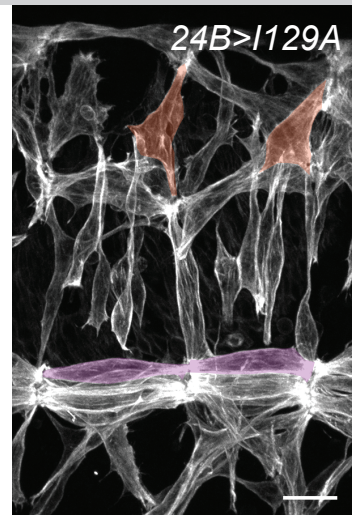


Figure 3. Bsd and Polo kinase are in a common myogenic pathway

A

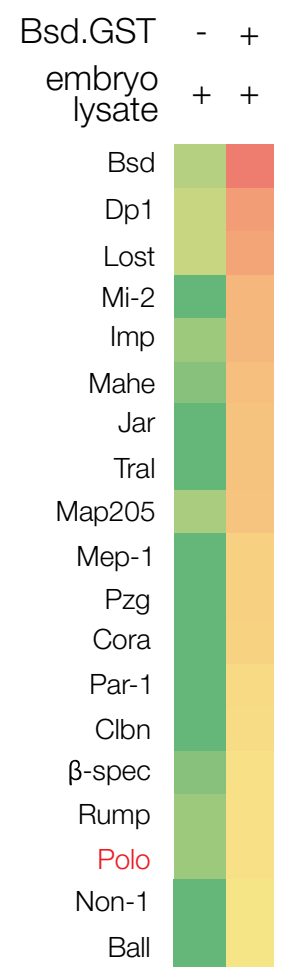

B

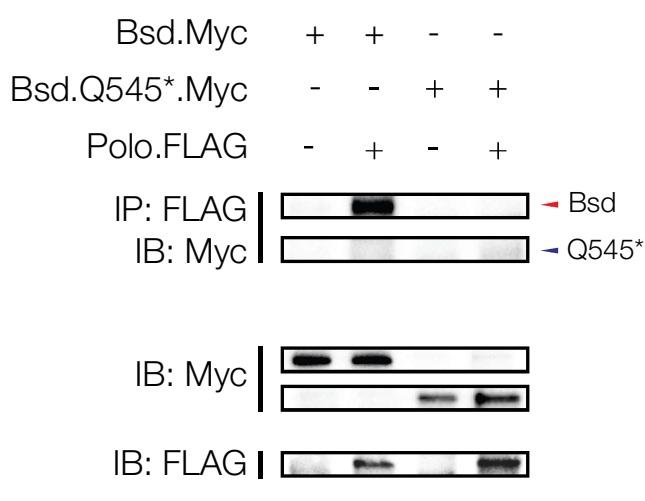

C

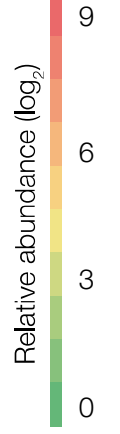

D

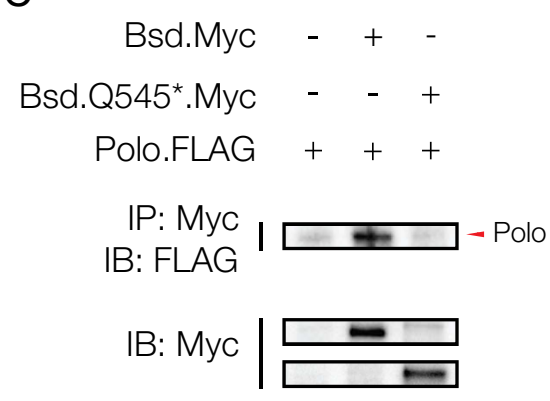

IB: FLAG |

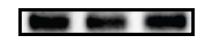

F

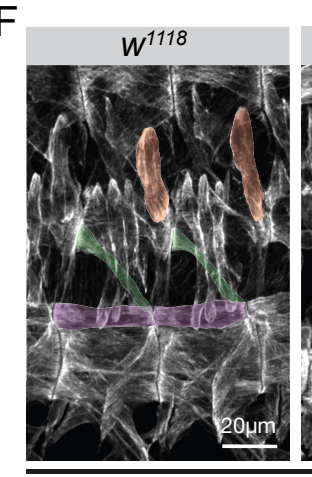

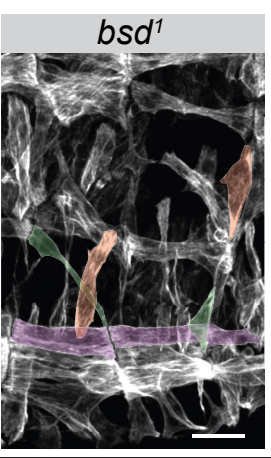

Tropomyosin

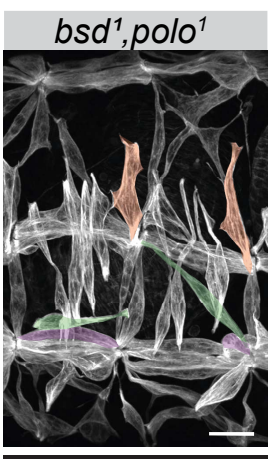

Tropomyosin

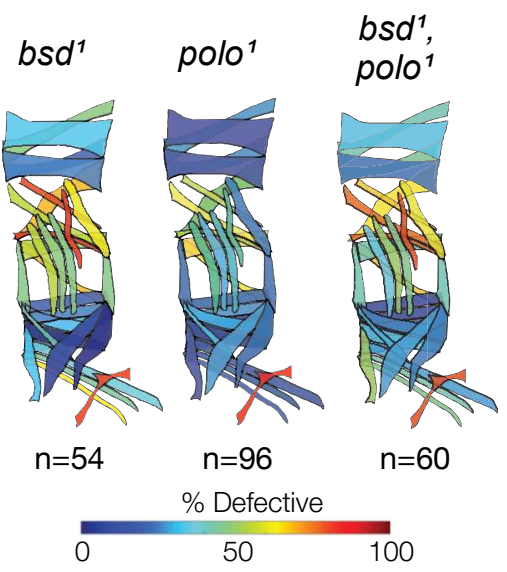

$\mathrm{H}$

G
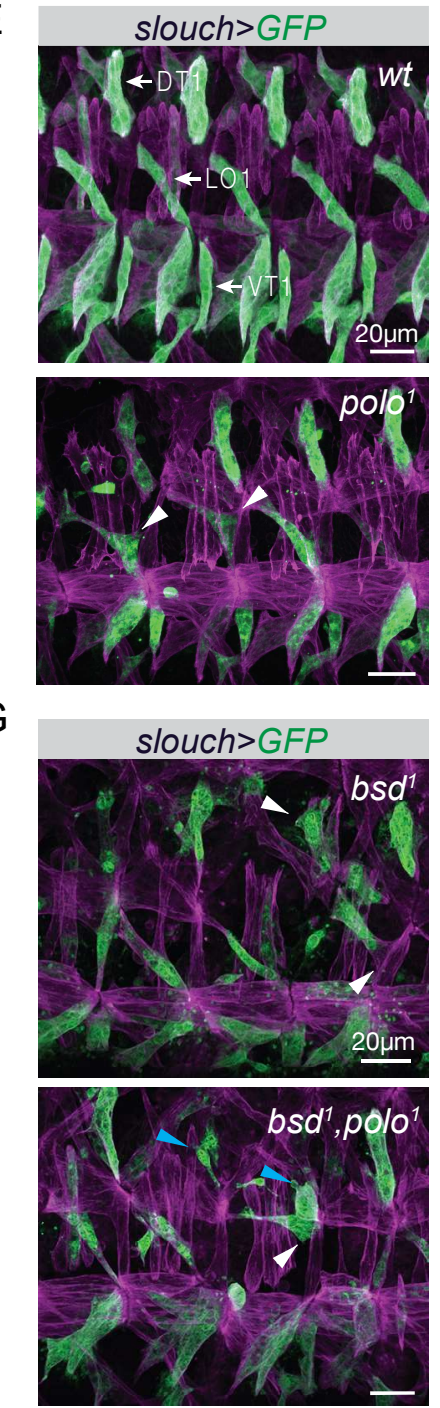

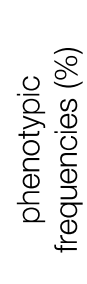

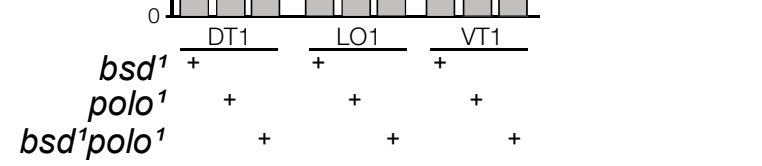


Figure 4. Bsd activates Polo to direct myotube guidance

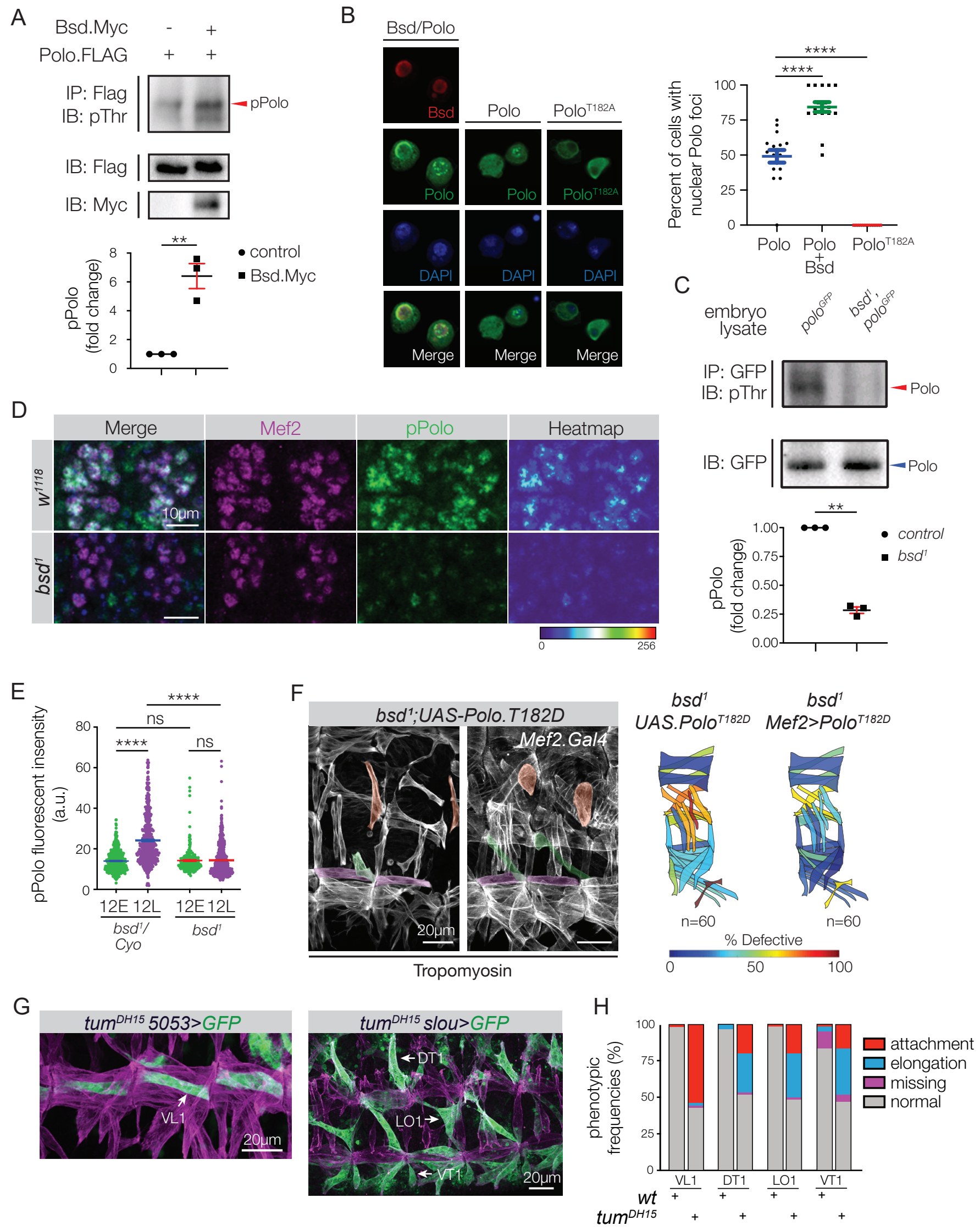


Figure 5. Bsd directs microtubule reorganization during myotube guidance

A

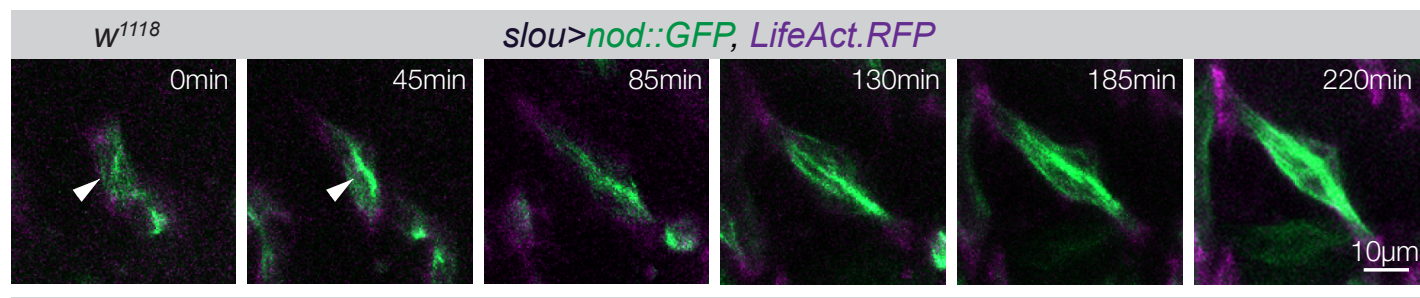

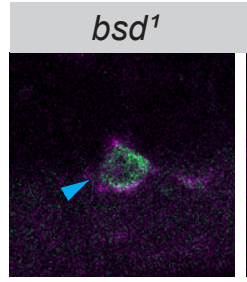

B
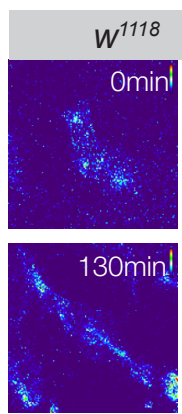

$b s d^{1}$
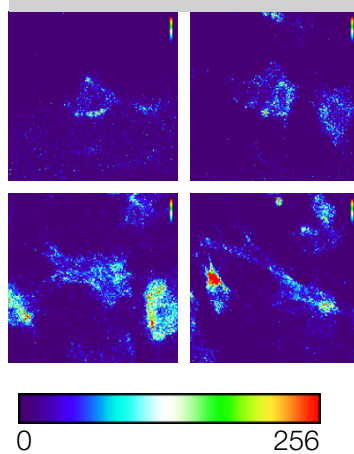

D
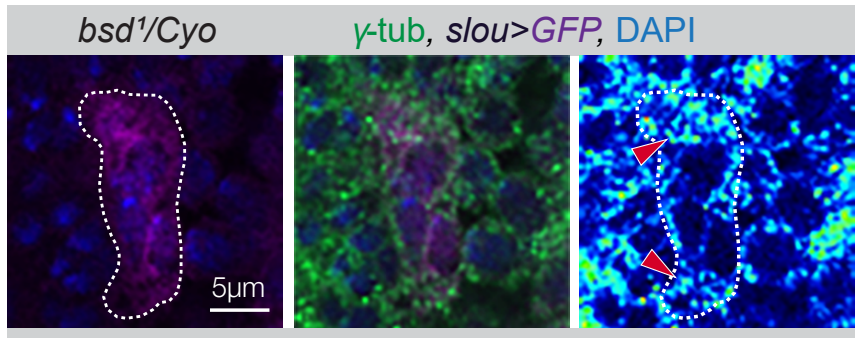

$$
b s d^{1}
$$
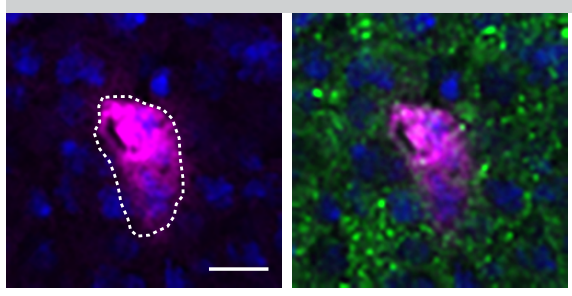
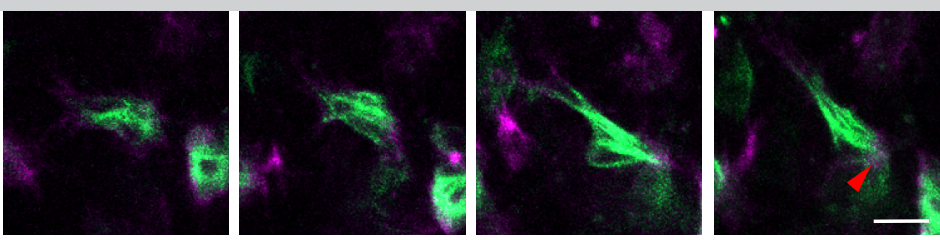

C

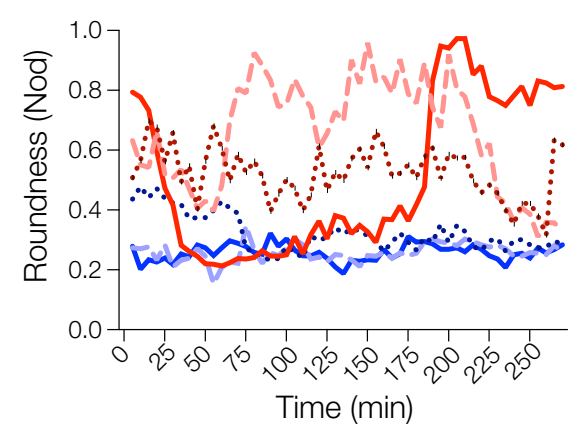

- $w^{1118} \# 1$

- $w^{1118} \# 2$

-.. $w^{1118} \# 3$

- bsd ${ }^{1} \# 1$

- $b s d^{1} \# 2$

-.. bsd $d^{1} \# 3$

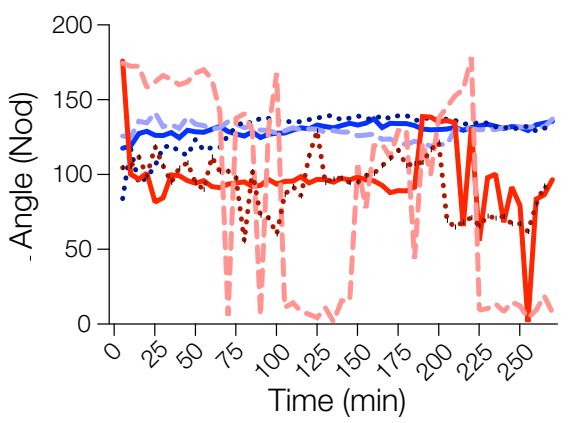

E

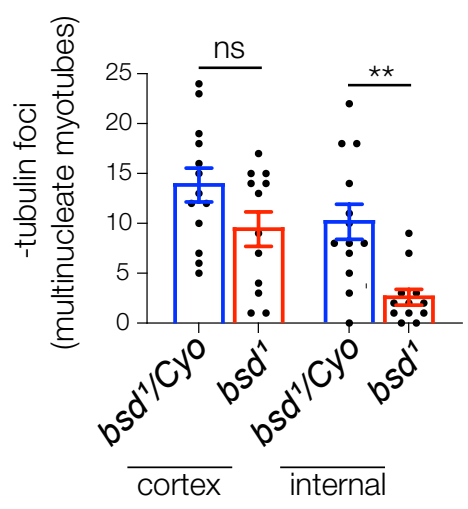


Figure 6. The Bsd orthologue Vrk3 is required for myotube elongation

A
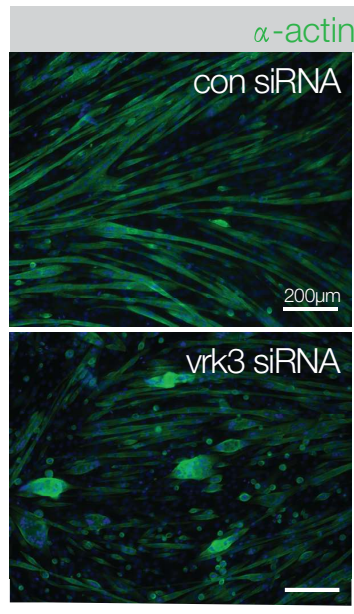

C

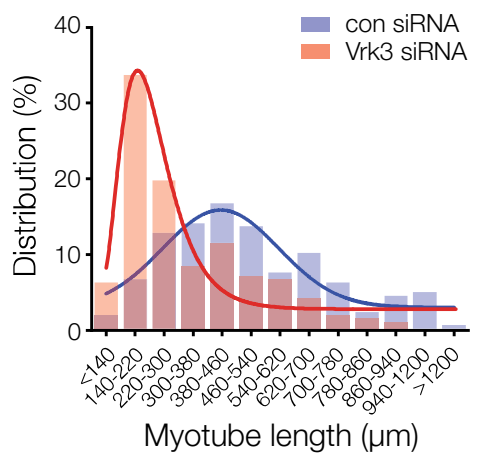

$\mathrm{F}$

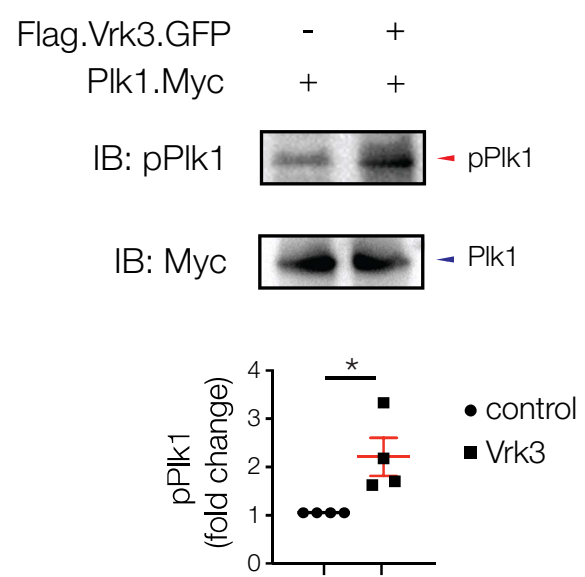

B

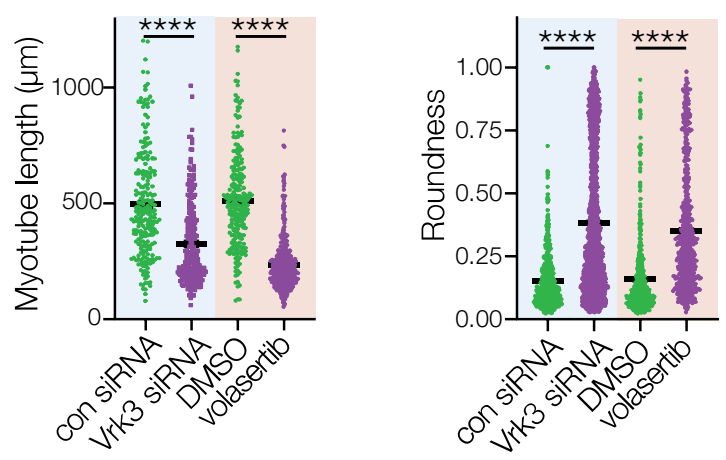

E

Flag.Vrk3.GFP $\quad-\quad+$

Flag.GFP $\quad+\quad-$

Plk1.Myc $\quad+\quad+$

IP: FLAG
IB: Myc I - Plk1

IB: Myc | - Plk1

IP: FLAG

G

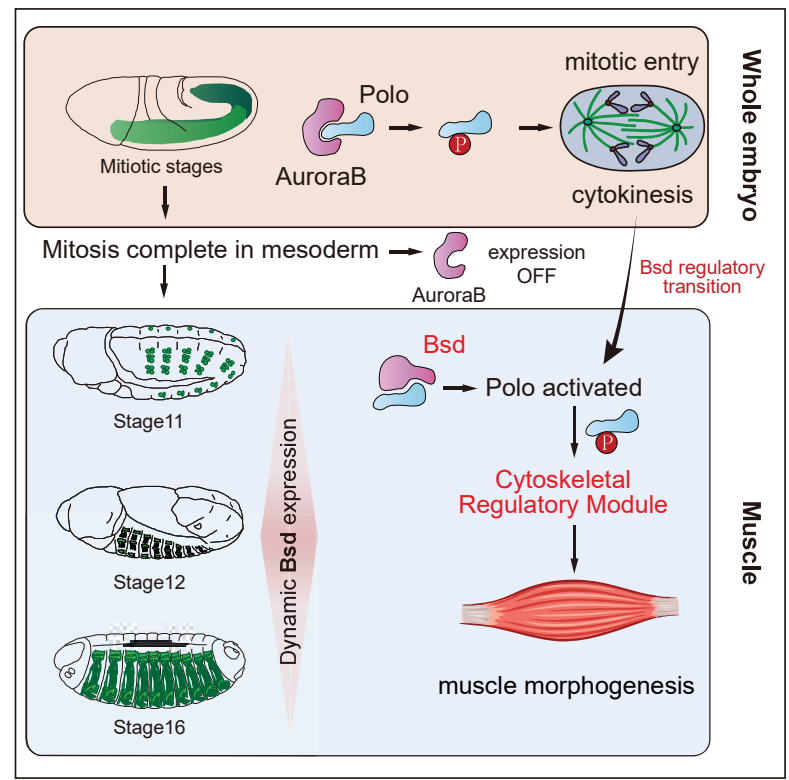


bioRxiv preprint doi: https://doi.org/10.1101/2020.06.16.155333; this version posted September 16, 2020. The copyright holder for this preprint (which was not certified by peer review) is the author/funder. All rights reserved. No reuse allowed without permission.

Figure S1, related to Figure 1

A

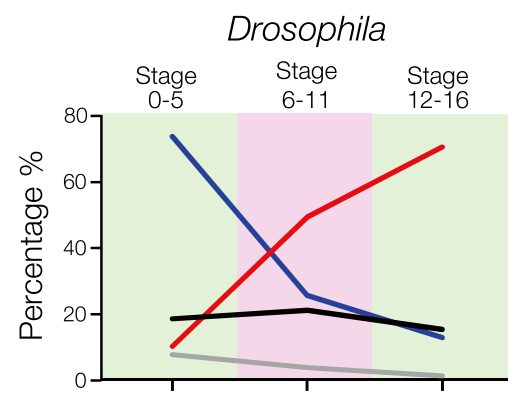

B

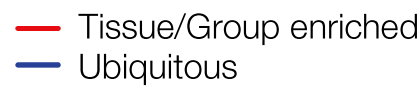

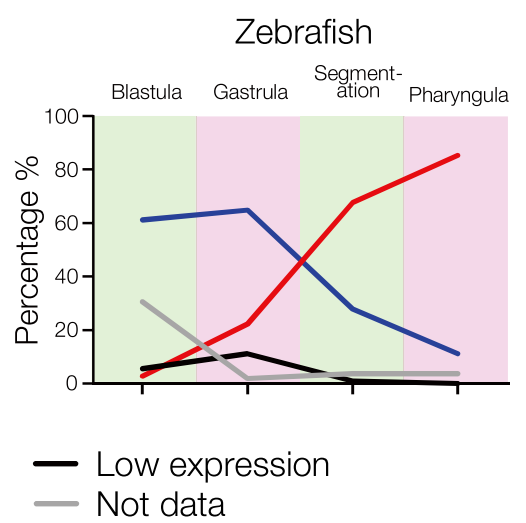

C

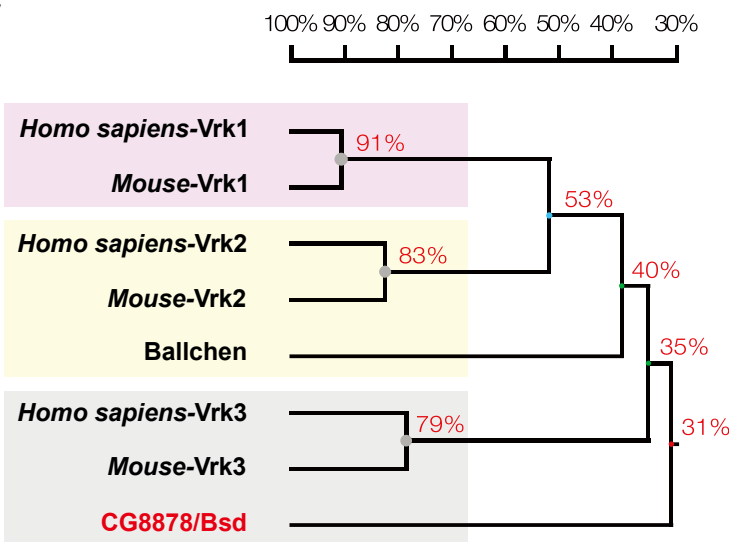

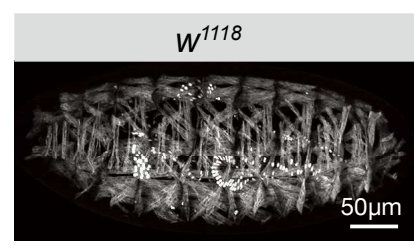

MHC.GFP

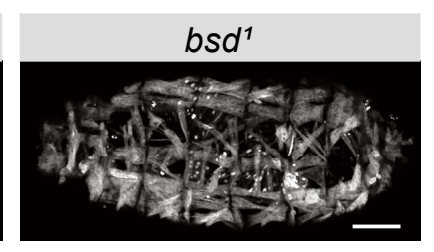

MHC.GFP

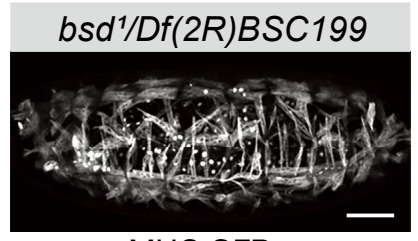

MHC.GFP

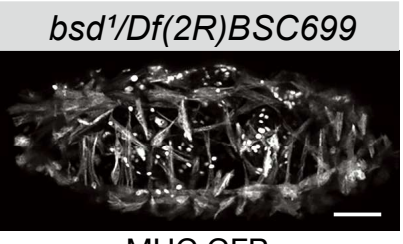

MHC.GFP

D

kinase domain _ ATP binding pocket _ linker region
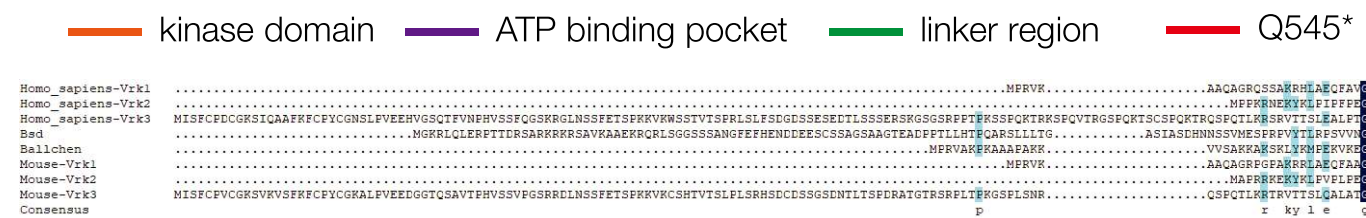

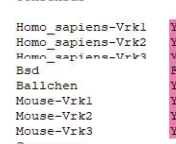
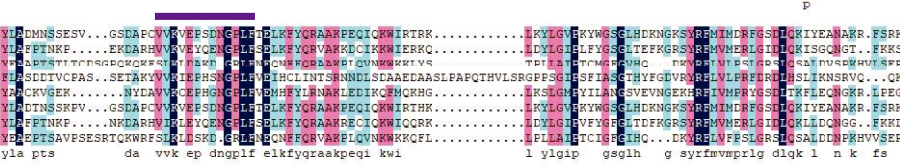

Homo_sapiens-Trk1
Homo_sapiens-Vrk2
Homo_sapiens-Vrk3

Homo_sapien
Bsa
Ballchen
Mouss-Vrk1
Mugse-Vrk2

Mouse-Vrk2
Mouse-Vrk3
Consensus

Homo_sapiens-Vrk1
Homo-sapiens-Vrk2
Homo-sabiens-Vrk3

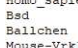

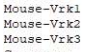

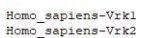

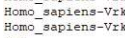

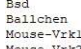

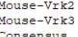

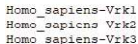

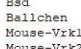

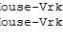

E

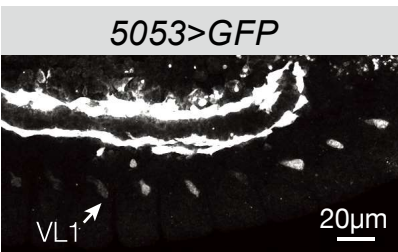

$b d^{1} d^{1}$ 053>GFP

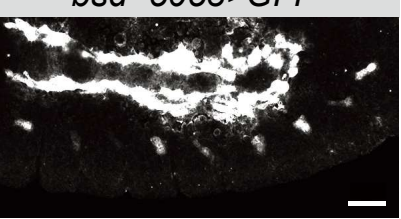

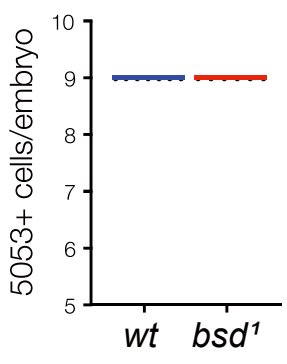

F

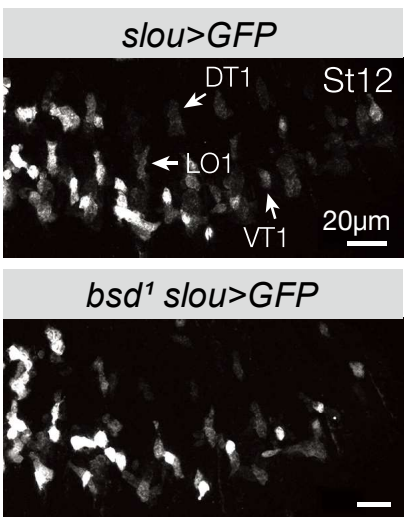

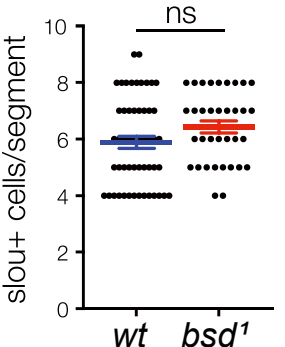


Figure S2, related to Figure 2

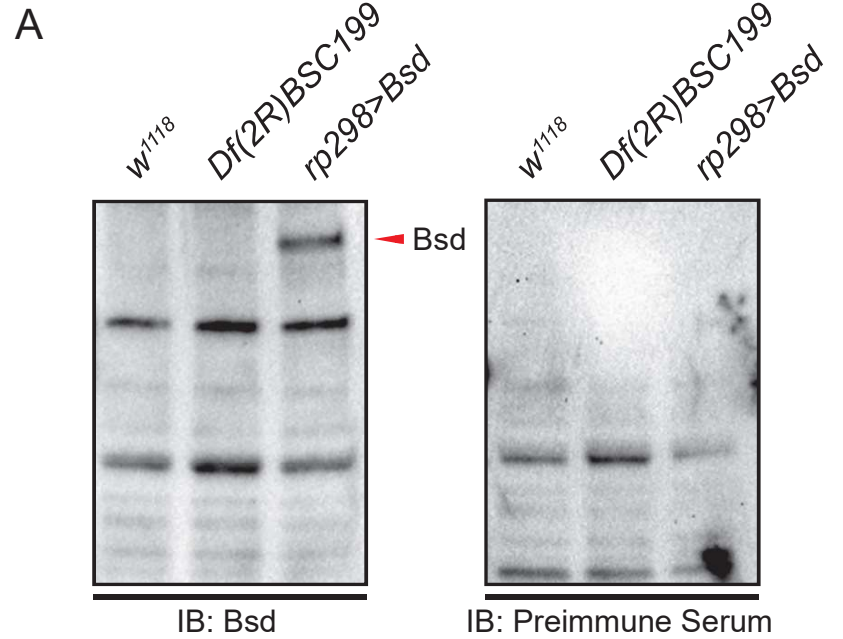

C

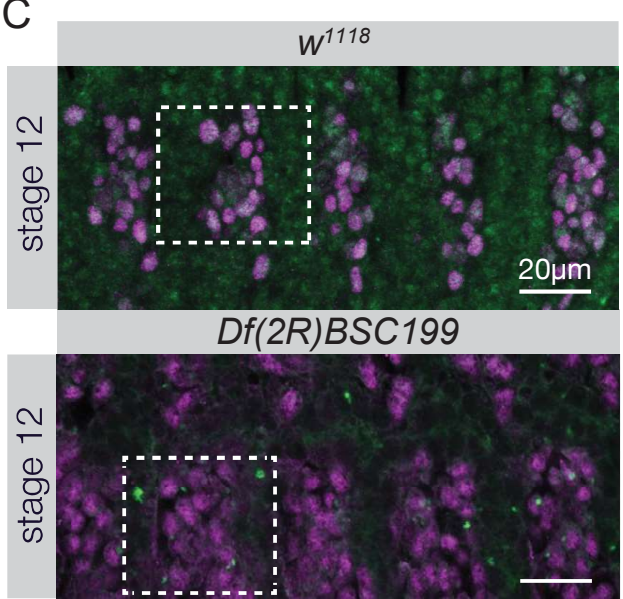

$\mathrm{D}$
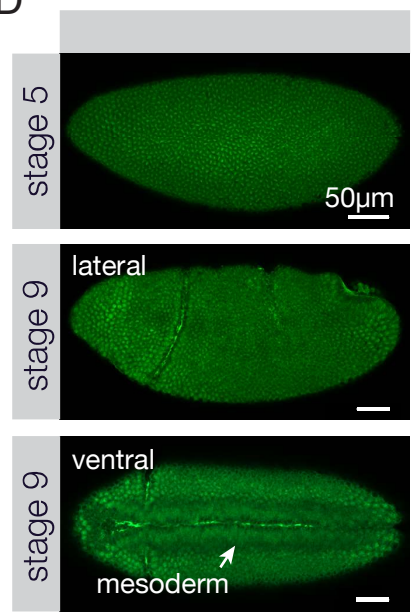

$\mathrm{E}$

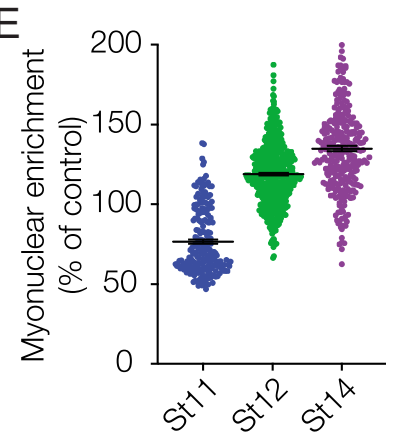

B
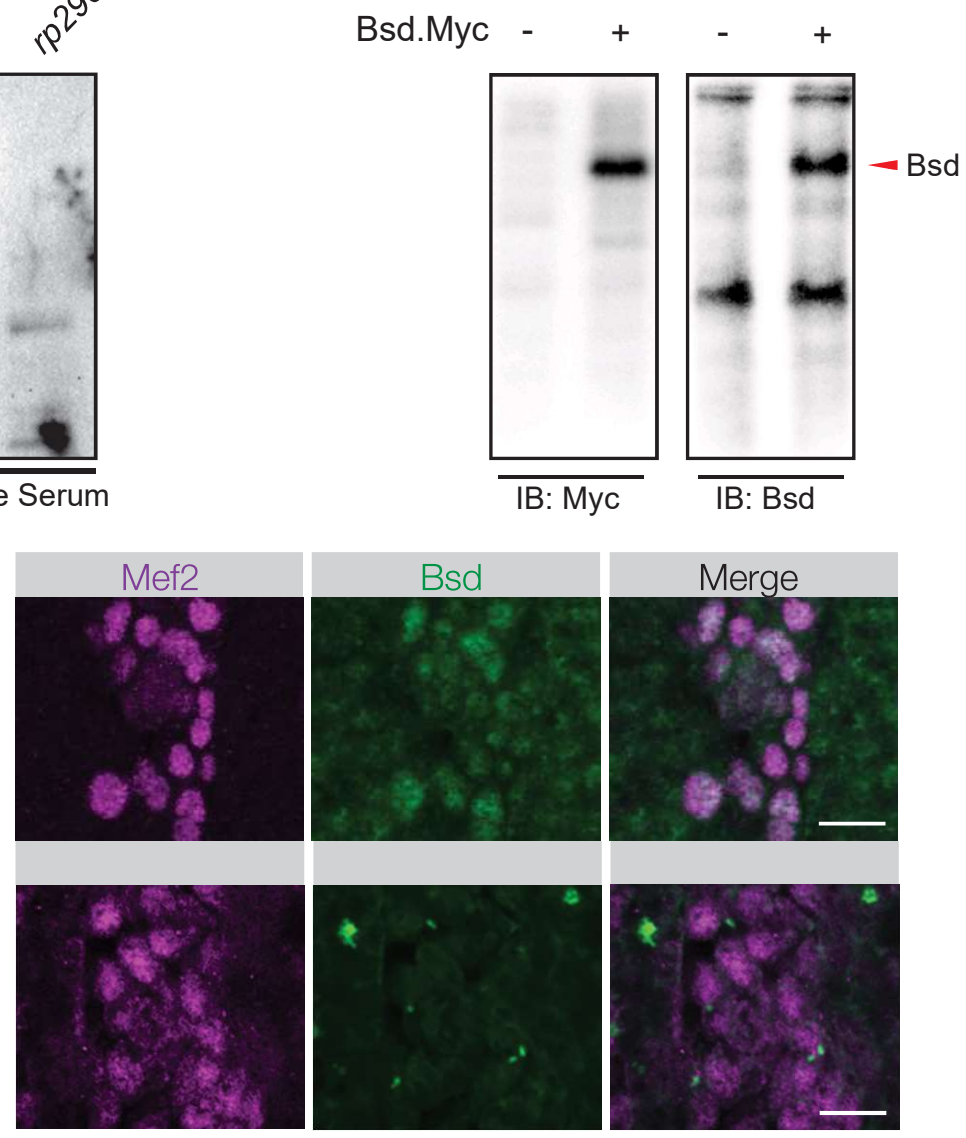
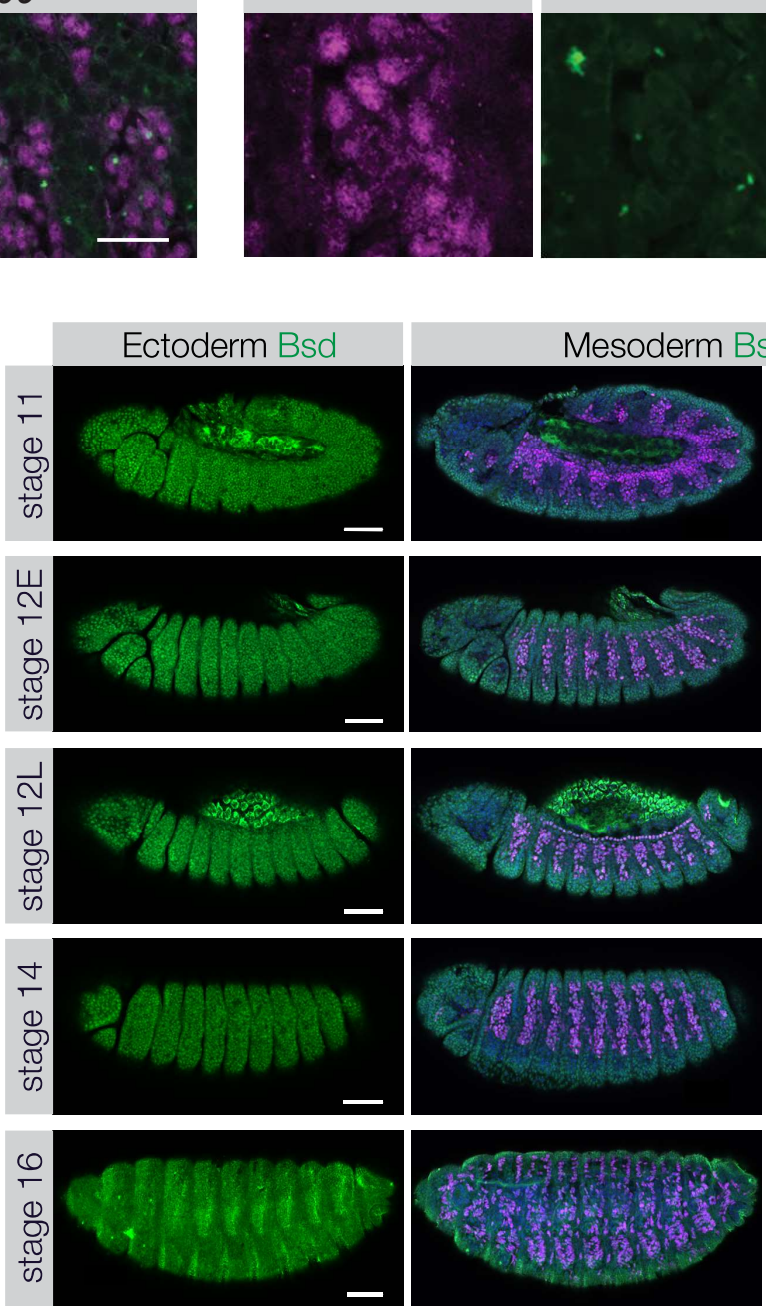
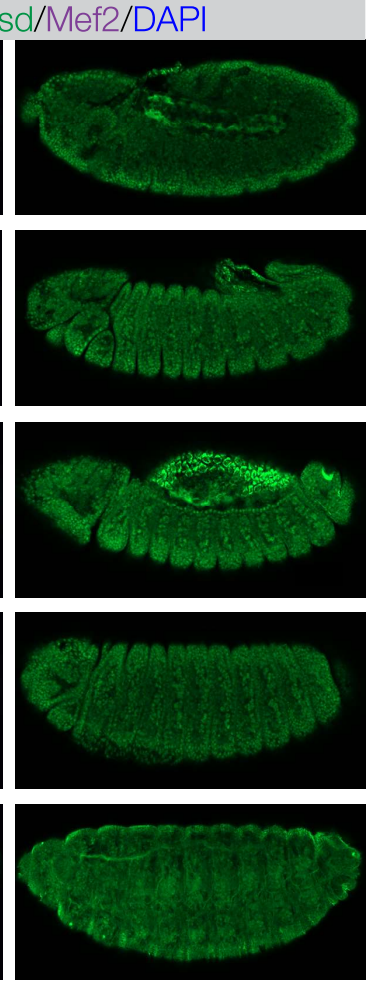
bioRxiv preprint doi: https://doi.org/10.1101/2020.06.16.155333; this version posted September 16, 2020. The copyright holder for this preprint (which was not certified by peer review) is the author/funder. All rights reserved. No reuse allowed without permission.

Figure S3, related to Figure 3

A

$\begin{array}{lllll}\text { GST-Bsd } & - & + & - & + \\ \begin{array}{c}\text { embryo } \\ \text { Iysate }\end{array} & - & - & + & +\end{array}$

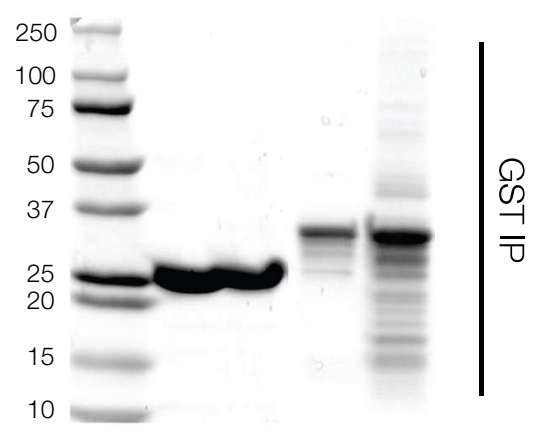

B
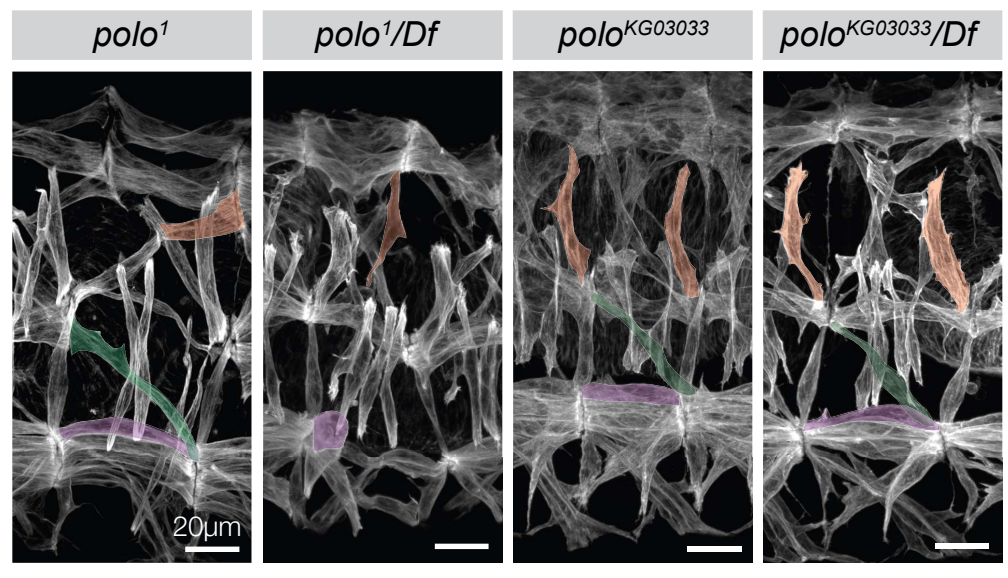

C
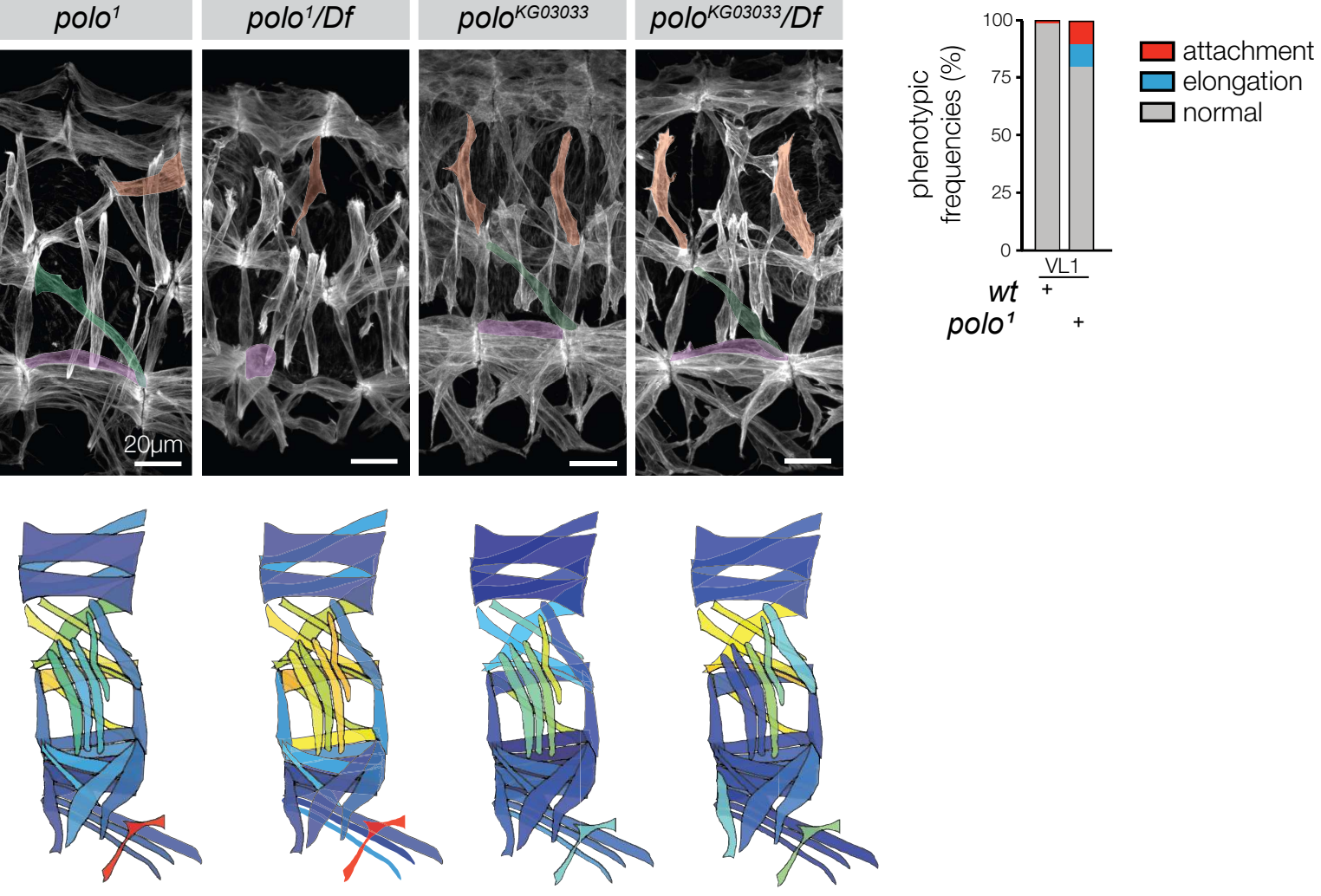

$\mathrm{n}=96$

$$
\begin{array}{cc}
n=36 & n=48 \\
\hline \text { \% Defective } & \\
\hline 50 & 100
\end{array}
$$

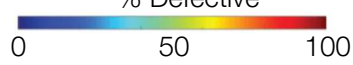


Figure S4, related to Figure 4

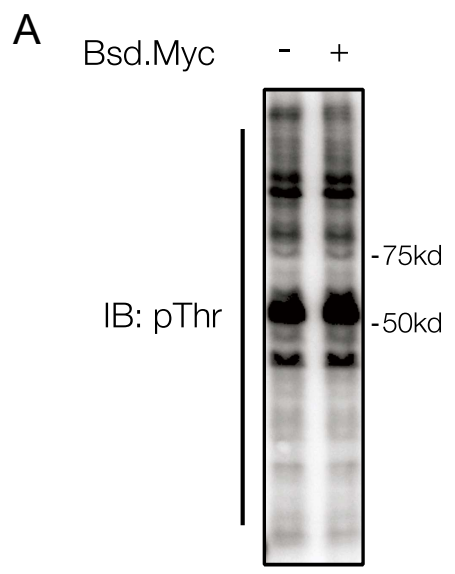

IB: Tub I

\section{C}

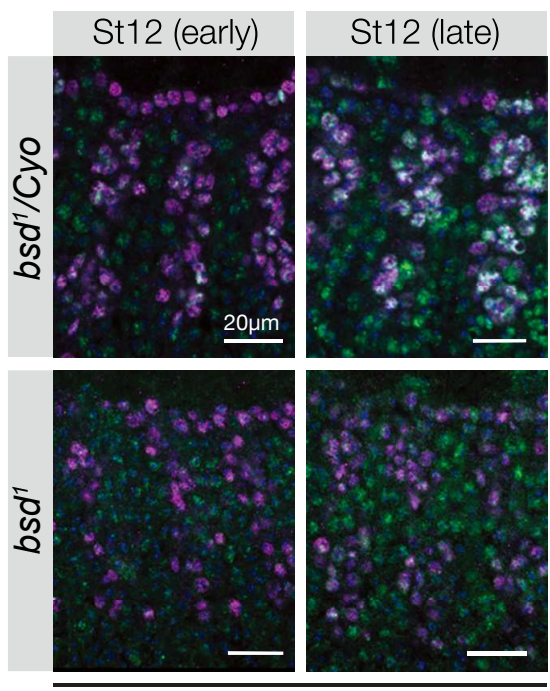

pPolo/Mef2

B
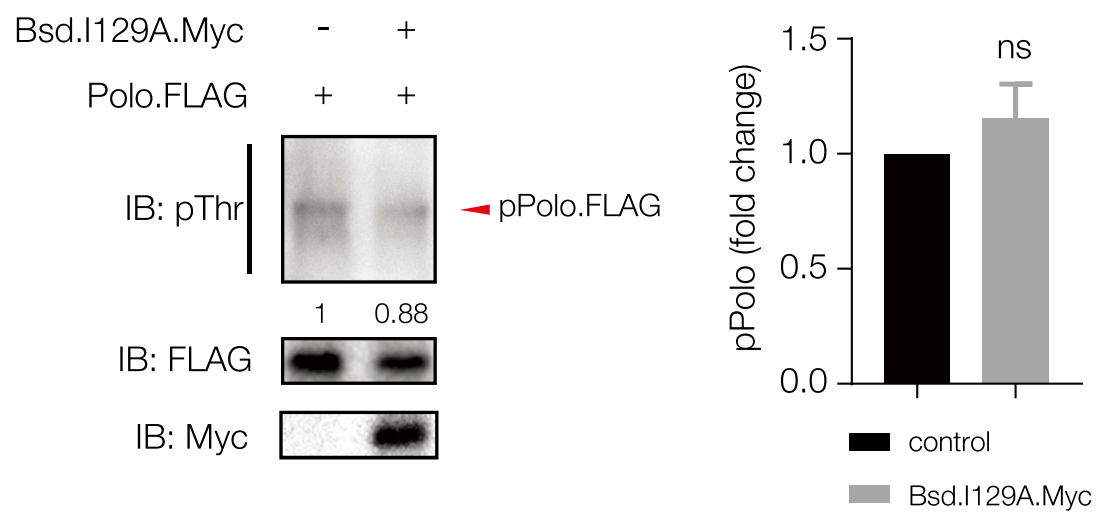

D
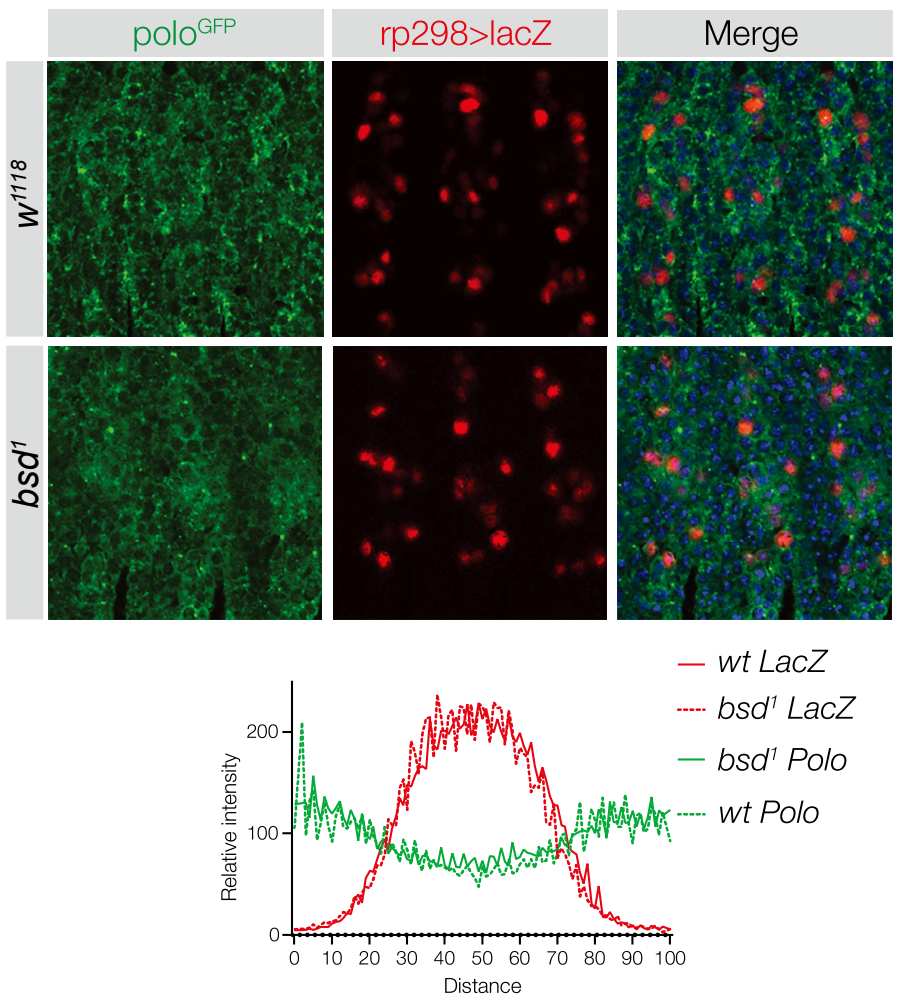
Figure S5, related to Figure 5

A

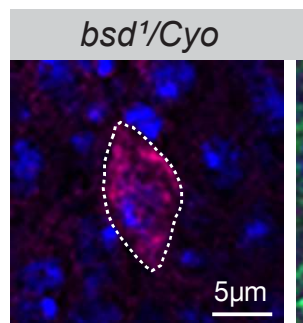

\section{$\gamma$-tub, slou> GFP, DAP}

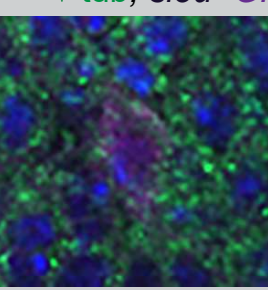

$b s d^{1}$

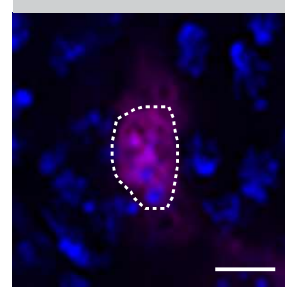

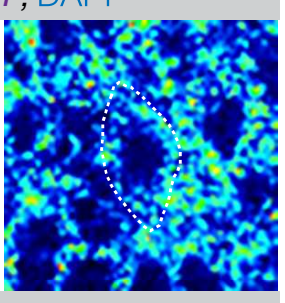

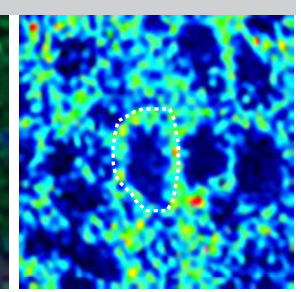

B

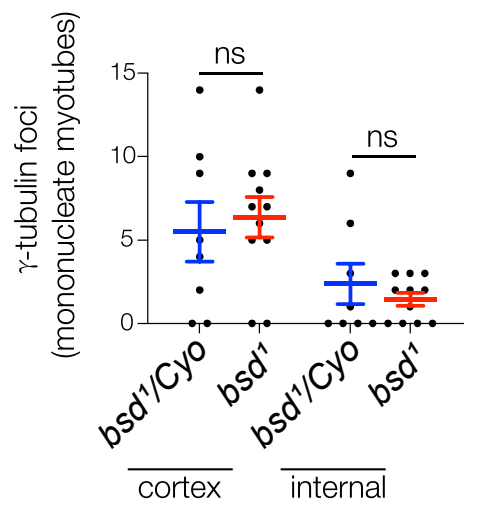




\section{Figure S6. Related to Figure 6}

A
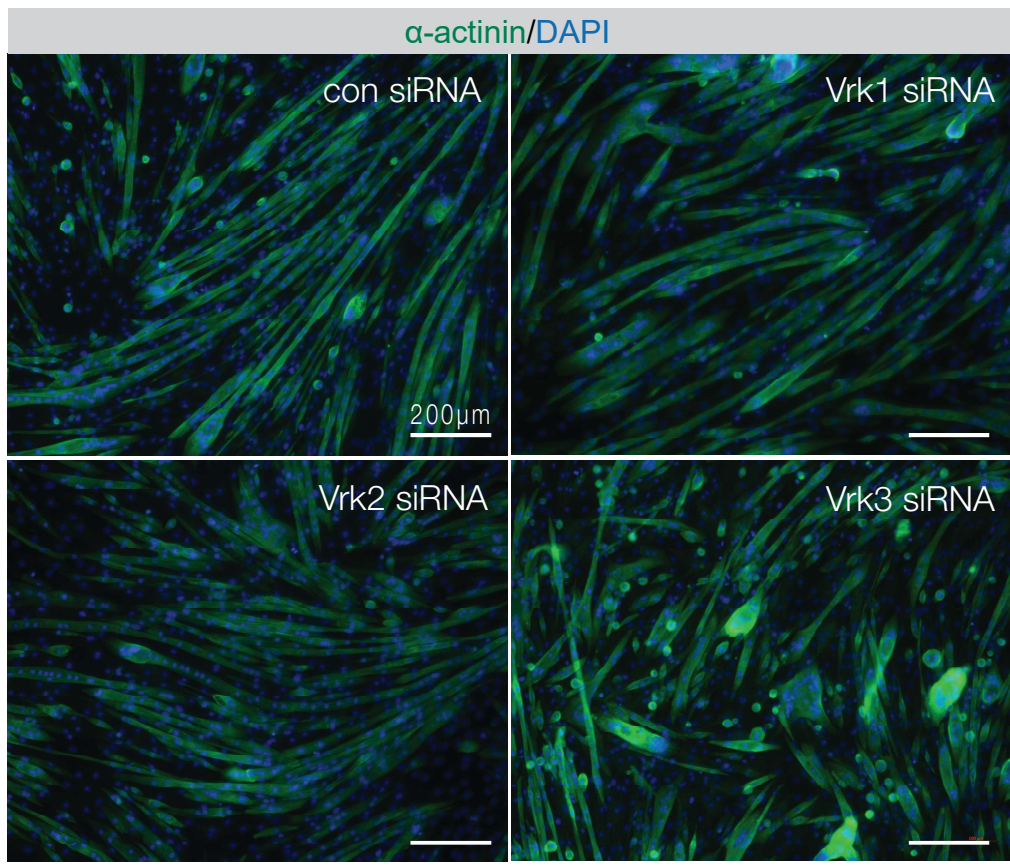

B

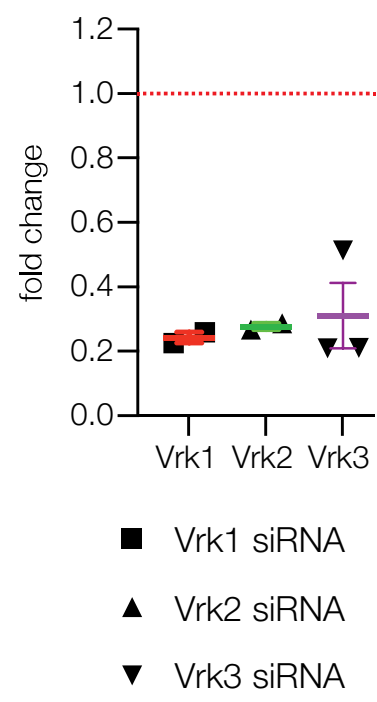

C
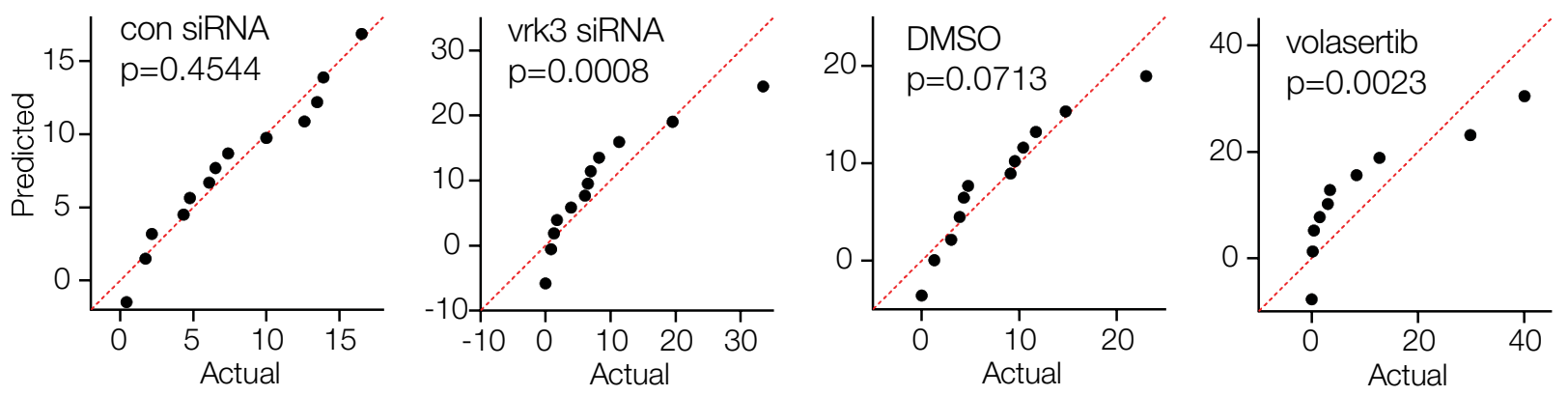

D

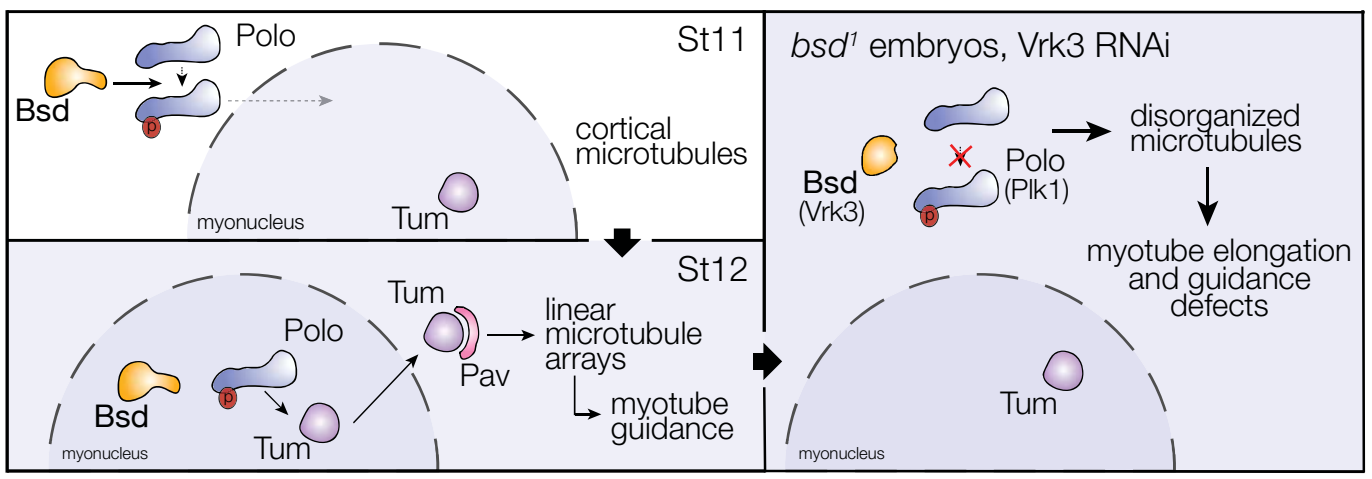

